\title{
Long-period Ap stars discovered with TESS data: The northern ecliptic hemisphere
}

\author{
G. Mathys ${ }^{1} \odot$, D. W. Kurtz ${ }^{2,3} \odot$, and D. L. Holdsworth ${ }^{3} \odot$ \\ 1 European Southern Observatory, Alonso de Cordova 3107, Vitacura, Santiago, Chile \\ e-mail: gmathys@eso.org \\ 2 Centre for Space Research, Physics Department, North West University, Mahikeng 2735, South Africa \\ 3 Jeremiah Horrocks Institute, University of Central Lancashire, Preston PR1 2HE, UK
}

Received 27 September 2021 / Accepted 30 December 2021

\begin{abstract}
The rotation periods of the magnetic Ap stars span five to six orders of magnitude. While it is well established that period differentiation must have taken place at the pre-main sequence stage, the physical processes that lead to it remain elusive. The existence of Ap stars that have rotation periods of tens to hundreds of years is particularly intriguing, and their study represents a promising avenue to gain additional insight into the origin and evolution of rotation in Ap stars. Historically, almost all the longest period Ap stars known have been found to be strongly magnetic; very few weakly magnetic Ap stars with very long periods have been identified and studied. To remedy that, we showed how a systematic search based on the analysis of TESS photometric data could be performed to identify super-slowly rotating Ap (ssrAp) stars independently of the strengths of their magnetic fields, with the intention to characterise the distribution of the longest Ap star rotation periods in an unbiased manner. We successfully applied this method to the analysis of the TESS 2-min cadence observations of Ap stars of the southern ecliptic hemisphere. For our present study, we applied the same approach to the analysis of the TESS 2-min cadence observations of Ap stars of the northern ecliptic hemisphere. We confirm that the technique leads to the reliable identification of ssrAp star candidates in an unbiased manner. We find 67 Ap stars with no rotational variability in the northern ecliptic hemisphere TESS data. Among them, 46 are newly identified ssrAp star candidates, which is double the number found in the southern ecliptic hemisphere. We confirm that super-slow rotation tends to occur less frequently in weakly magnetic Ap stars than in strongly magnetic stars. We present new evidence of the existence of a gap between $\sim 2 \mathrm{kG}$ and $\sim 3 \mathrm{kG}$ in the distribution of the magnetic field strengths of long period Ap stars. We also confirm that the incidence of roAp stars is higher than average in slowly rotating Ap stars. We report the unexpected discovery of nine definite and five candidate $\delta$ Sct stars, and of two eclipsing binaries. This work paves the way for a systematic, unbiased study of the longest period Ap stars, with a view to characterise the correlations between their rotational, magnetic, and pulsational properties.
\end{abstract}

Key words. stars: chemically peculiar - stars: magnetic field - stars: rotation - stars: oscillations

\section{Introduction}

It is now well established that 5 to $10 \%$ of all stars of spectral types $\mathrm{O}$ to early $\mathrm{F}$ that have radiative atmospheres host strong, predominantly dipolar large-scale organised magnetic fields (Grunhut et al. 2017; Schöller et al. 2017; Sikora et al. 2019a). Among these magnetic early-type stars, it is convenient to distinguish three groups in order of increasing temperature: the chemically peculiar A and B stars (Ap and Bp stars, which are often collectively referred to simply as Ap stars), the magnetic early B stars (with spectral types ranging from B5 to B0), and the magnetic $\mathrm{O}$ stars. The distribution of the dipole field strengths is to first order similar for all three types (Shultz et al. 2019a).

Furthermore, the cumulative distributions of the rotation periods of the Ap, magnetic early $\mathrm{B}$, and magnetic $\mathrm{O}$ stars are also similar to first order (Shultz et al. 2018). All of these stars rotate, on average, more slowly than their non-magnetic counterparts with similar temperatures, and their rotation periods span five to six orders of magnitudes, from $\sim 0.5 \mathrm{~d}$ to at least $\sim 300 \mathrm{yr}$, and possibly as much as $\sim 1000 \mathrm{yr}$ (Mathys 2017). Although recent observations suggest that rotation slows down over time in the more massive stars that have higher mass loss, which is qualitatively consistent with the occurrence of magnetospheric braking (Shultz et al. 2019a), such a loss of angular momentum on the main sequence is at most marginal for Ap stars (Kochukhov \& Bagnulo 2006; Hubrig et al. 2007). Since evolutionary changes to the rotation periods of Ap stars during their main-sequence lifetimes are small, most of the period differentiation must have occurred at the pre-main sequence stage. Accordingly, consideration of the present distribution of the rotation periods provides valuable constraints towards the theoretical understanding of the physical mechanisms responsible for this differentiation.

Even if the rotation rates of higher mass magnetic stars evolve more on the main sequence than those of Ap stars, the arguments presented by Mathys et al. (2020, hereafter Paper I) to support the value of studying Ap stars for understanding the rotational properties of the whole population of magnetic early-type stars remain valid. Many more Ap stars are known than magnetic early B and O stars; Ap stars as a group have been studied for much longer than the other magnetic early-type stars, and they lend themselves better than the latter to the study of rotational properties. However, the present knowledge of the slow-rotation tail of their period distribution remains incomplete. 
In particular, the occurrence of extremely slow rotation in weakly magnetic Ap stars is poorly characterised (as discussed in Sect. 4). This results, at least in part, from observational biases (Paper I), but may also reflect actual differences in the distribution of the rotation rates of stars with different magnetic field strengths. Establishing if the distribution of the rotation periods is similar or not for the weakly and strongly magnetic stars represents an essential element for the theoretical understanding of the rotational behaviour of Ap stars.

In Paper I, we showed how TESS data can be exploited to carry out a systematic search for long-period Ap star candidates, in a way that is fully independent of their magnetic field strengths. Following Mathys (2020), we define long period to be greater than $50 \mathrm{~d}$, and we refer to these stars as 'super slowly rotating Ap (ssrAp) stars'. The approach that is used for this search takes advantage of the fact that Ap stars are oblique rotators, with a nonuniform surface brightness whose distribution, which is stable over timescales much longer than the rotation periods, presents a certain degree of symmetry about the predominantly dipolar component of the magnetic field. As the latter is, in general, inclined with respect to the rotation axis, rotationally induced periodic photometric variations are observed, with typical amplitudes of a few hundredths of a magnitude.

Most of the Ap stars observed with TESS clearly show such low-frequency ( $v_{\text {rot }} \lesssim 2 \mathrm{~d}^{-1} ; P_{\text {rot }} \gtrsim 0.5 \mathrm{~d}$ ) photometric variations due to rotational modulation, sometimes together with superimposed high-frequency photometric variations caused by pulsation. See Paper I for some examples. However, some do not show any low-frequency variations over one, or several, TESS 27-d sector(s). Almost all of those are slow rotators, with periods $P_{\text {rot }} \gtrsim 27 \mathrm{~d}$. The only exceptions are those stars for which the inclination of the rotation axis to the line of sight is too small $\left(i \lesssim 5^{\circ}\right.$ ) or the obliquity of the magnetic axis with respect to the rotation axis is too small $\left(\beta \lesssim 5^{\circ}\right)$. As discussed in Paper I, under the assumption of random distribution of the inclinations and obliquities, stars for which one or both of these conditions are fulfilled should be rare, representing less than $1 \%$ of all Ap stars. Admittedly, the distribution of the magnetic obliquities has not yet been uniquely constrained. The results of some works suggest that it may not be random, although their conclusions are discrepant, while other recent studies yield results consistent with random obliquities (see Paper I for more details).

Paper I was devoted to the analysis of the TESS 2-min cadence observations of the Ap stars from the southern ecliptic hemisphere (TESS Sectors 1-13). Of the 1014 stars for which TESS SPOC (Science Processing Operations Center; Jenkins et al. 2016) data were available, there were 60 for which no low-frequency photometric variations were observed. For 31 of them, existing constraints on the rotation period and/or $v \sin i$ are consistent with (very) slow rotation. For only six of the nonvariable stars, the available spectroscopic observations showed broad spectral lines indicative of short rotation periods. These are probably (relatively) fast rotators with nearly aligned magnetic and rotation axes. Their rate of occurrence is compatible with the expected one for low obliquity $\left(\beta \lesssim 5^{\circ}\right)$. The 23 remaining non-variable stars, whose rotation periods are unknown and for which no high-resolution spectra have been obtained, are newly identified long-period candidates. Spectroscopic confirmation of their low $v \sin i$ will further establish the validity of the search technique introduced in Paper I.

Hereafter, we apply this technique to the analysis of the TESS 2-min cadence SPOC data of the Ap stars from the northern ecliptic hemisphere (TESS Sectors 14-26).

\section{TESS data}

The sample of stars that was analysed consists of 492 stars that were classified as, or suspected to be, Ap stars that had 2-min cadence data available. By running an automated detection algorithm (see Paper I for details) on this sample, a preliminary list of 188 stars that did not show definitive photometric variations over a time scale of $27 \mathrm{~d}$ (or longer when more than one sector is available) was established. In a second step, this list was critically reviewed to restrict it to bona fide Ap stars. To this effect, we used as a primary reference the Catalogue of Ap, HgMn and Am stars (Renson \& Manfroid 2009). We excluded from our list those stars that are not present in the Catalogue with an Ap spectral classification, as well as those stars whose peculiar nature is flagged as doubtful ('?' in the first column in the Catalogue) or that have been improperly considered to have an Ap nature ("/, in the first column). However, in a second step, we reinstated in the list six (mostly faint) stars that are not in the Catalogue but were subsequently identified as definite rapidly oscillating Ap (roAp) stars. Then, we carried out a literature survey using the SIMBAD Astronomical Database to check for possible cases of stars from the Catalogue whose Ap nature had been disproved in studies published more recently. We also removed these stars from the list, which as a result was reduced to 95 entries.

As a final selection step, the TESS data obtained for the 95 remaining stars were inspected manually to confirm the probable lack of low-frequency variability diagnosed by application of the detection procedure. This led to the elimination of another 28 stars, whose light curves are similar to the typical rotational light curves of short-period $\left(P_{\text {rot }}<27 \mathrm{~d}\right)$ Ap stars. The final list, which contains 67 long-period Ap star candidates, is presented in Table A.1.

Amplitude spectra for some of the stars that we have identified as probable long rotation period Ap stars are shown in Fig. 1. The amplitude spectra of the other long-period Ap star candidates are shown in Appendix B. The ordinate axis was chosen to have a maximum amplitude of 0.4 mmag for most stars to show easily the lack of a low frequency peak associated with rotation in Ap stars at $v_{\text {rot }} \leq 2 \mathrm{~d}^{-1}$. In the left column the maximum of the abscissa was chosen to be $5 \mathrm{~d}^{-1}$ to encompass the low frequency range of Ap rotation, to show some additional range so it can be seen that the noise settles to be white by $5 \mathrm{~d}^{-1}$, and to show the typical harmonic series for Ap stars that do show rotation. Of course, those examples are not in the figures of this papersince they do not show rotational variation - but they were part of the selection process. The right column has a maximum for the abscissa of $360 \mathrm{~d}^{-1}$, which is the Nyquist frequency for the TESS 2-min data. These plots show that the higher frequency noise is white, that the highest noise peaks are usually (except for fainter stars) under $0.05 \mathrm{mmag}$, and they show $\delta$ Sct, $\gamma$ Dor, and roAp pulsation, where it is present.

The list of Ap stars from the northern ecliptic hemisphere (TESS Sectors 14-26) that show no rotational variation in the TESS data contains 67 entries. These 67 stars are likely to have rotation periods longer than $27 \mathrm{~d}$.

Eight of these stars show resolved magnetically split lines: seven were listed in Table 1 of Mathys (2017); the eighth one (BD+44 3063) was reported by Balona et al. (2011). Of the stars with resolved magnetically split lines that appear in Table A.1, the latter is the only one whose rotation period is not accurately known. Furthermore, the list includes seven stars whose unresolved spectral lines appear sharp, or rather sharp, in our highresolution AURELIE spectra. Additionally, there are three more stars, HD 17330, HD 92728, and HD 96003, with line shapes 
G. Mathys et al.: Long period Ap stars
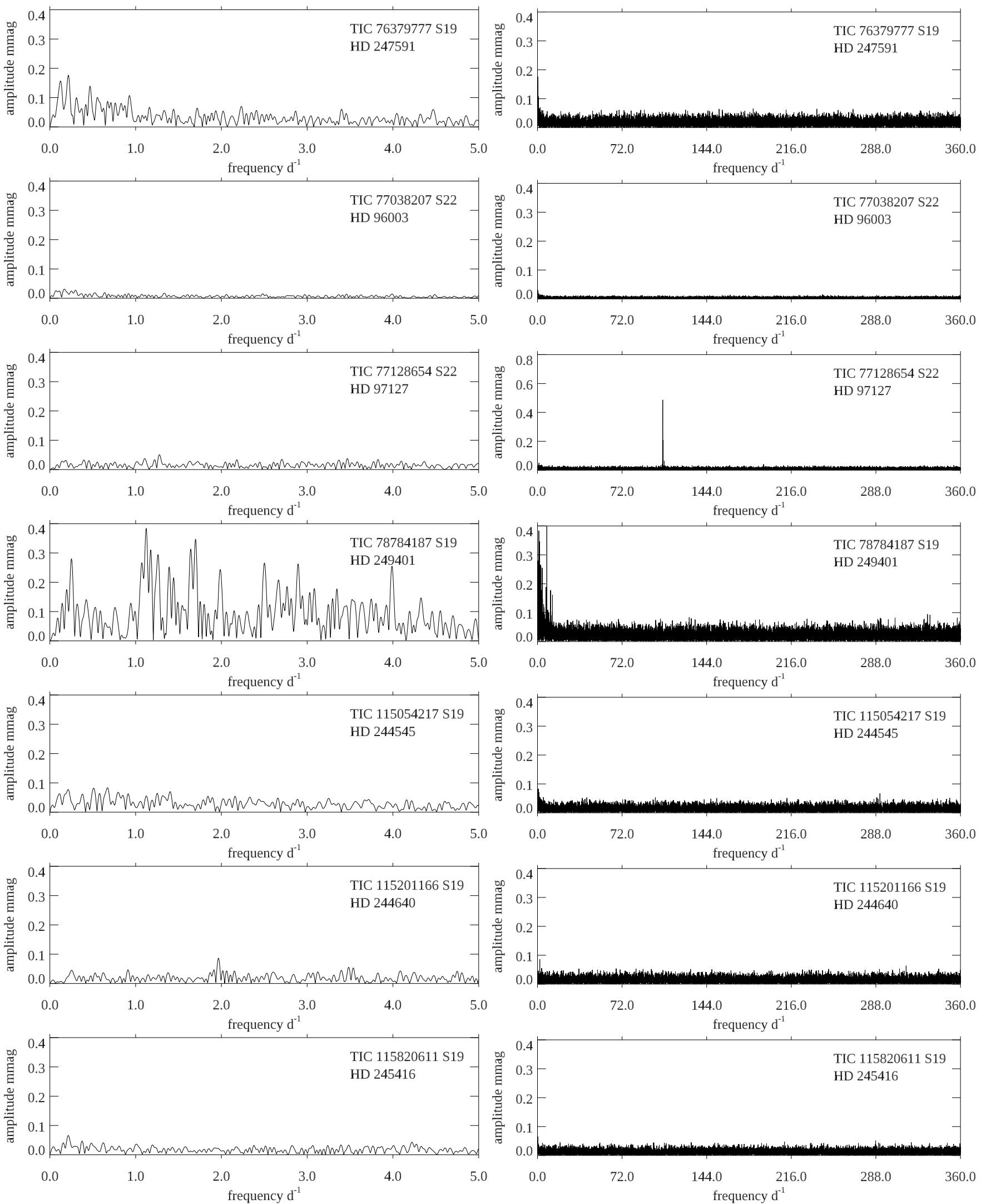

Fig. 1. Amplitude spectra for the long-period Ap stars. Each row presents a low-frequency amplitude spectrum showing no rotational variation in the left panel, and a full amplitude spectrum to the Nyquist frequency of $360 \mathrm{~d}^{-1}$ in the right panel, which allows detection of $\delta$ Sct or roAp pulsation. We note the occasional changes of ordinate scale to accommodate pulsation peaks. TIC 77128654 (HD 97127) is an roAp star (Sect. 2.1). TIC 78784187 (HD 249401) is a possible $\delta$ Sct star. 
that do not significantly differ from the instrumental profile in medium-high resolution spectra according to published information, which indicates an upper limit of $\sim 20 \mathrm{~km} \mathrm{~s}^{-1}$ for $v \sin i$.

Accurate periods of variation are available in the literature for 14 of the stars of our list. For eleven of them, the periods are definitely longer than $\sim 27 \mathrm{~d}$. The three exceptions are HD 22860 , for which we cannot exclude a short period, albeit inconsistent with the published value (Wraight et al. 2012), HD 89069, for which there is no evidence in the TESS data of the value of the period reported by Netopil et al. (2017), and HD 148330, for which the value derived by Ziznovsky \& Romanyuk (1990) may be spurious, as the amplitude of the photometric variations from which it is determined, $\sim 0.02$ mag peak-to-peak, is inconsistent with the lack of variability of the TESS data. For three more of the stars whose periods are accurately known, the values are less than $50 \mathrm{~d}$ : HD $14437\left(P_{\text {rot }}=26.87\right)$, HD 12288 $\left(P_{\text {rot }}=34\right.$ d 9$)$, and HD $335238\left(P_{\text {rot }}=48.7\right)$. These three stars are not strictly speaking ssrAp stars as they do not fulfil the criterion of $P_{\text {rot }}>50 \mathrm{~d}$ defined by Mathys (2020). However, this threshold is arbitrary, and unrelated to the detection threshold of the present search, which is set by the 27-d length of a TESS sector. The published periods are consistent with the lack of variability over a 27-d TESS sector. The three stars considered are definitely slow rotators, albeit not so extreme as many others.

At least some magnetic field measurements exist for 17 of the 67 stars of Table A.1. Eight of the 17 stars have resolved magnetically split lines; seven have sharp, unresolved lines; and for two, HD 17330 and HD 96003, $v \sin i$ is at most of the order of the instrumental profile width $\left(20 \mathrm{~km} \mathrm{~s}^{-1}\right)$.

Out of the 67 stars of the list, five are roAp, and eight more may also be. Assuming that they can be confirmed, $19 \%$ of the ssrAp candidates of the present survey would be roAp stars - less than the $22 \%$ found among the ssrAp candidates of the southern ecliptic hemisphere survey, but still considerably more than the overall occurrence rate among all the southern ecliptic hemisphere Ap stars observed by TESS (Paper I).

Unexpectedly, nine of the 67 stars of Table A.1 are definite $\delta$ Scuti stars, and five more may also be. This is unusual for Ap stars. Furthermore, two stars may be part of eclipsing binaries also a rare occurrence among Ap stars. We look at these special cases in more detail in the coming sections.

\subsection{The roAp stars}

Our goal is to understand the origin and impact of super slow rotation in Ap stars. Our discovery of an apparently high incidence of roAp stars amongst the ssrAp stars suggests that super slow rotation may be conducive to, but not necessary for, pulsation driving in roAp stars. This is an unanswered question that our studies of ssrAp stars will address. We therefore assess the roAp stars individually here. The roAp candidates typically have a single peak in the roAp pulsation frequency range with at least a $5 \sigma$ confidence in amplitude.

\subsubsection{TIC 26749633, KIC 11031749}

TIC 26749633 is a roAp star discovered by Hey et al. (2019) in Kepler long cadence data using superNyquist asteroseismology (Murphy et al. 2013). They found a single, unmodulated frequency at $118.6 \mathrm{~d}^{-1}$ with an amplitude of only $26 \mu \mathrm{mag}$. It is therefore unsurprising that we do not detect this pulsation in the TESS S14-15 data (Fig. B.1), as the highest noise peaks are about $180 \mu \mathrm{mag}$ as a consequence of the relative faintness of this star $(V=12.50)$. Hey et al. (2019) did find evidence of binarity using the phase modulation (PM) technique (Murphy et al. 2018). They obtained an orbital period of $1036 \mathrm{~d}$ and a mild eccentricity of $e=0.2$. The mass function gives $M_{2} \sin i=$ $0.1 M_{\odot}$, indicating a lower main-sequence companion. Visual binaries are known among the roAp stars (Schöller et al. 2012), including some of the brightest and well-known examples, such as $\alpha$ Cir, $\gamma$ Equ, and HR 3831. For TIC 26749633 an orbital period of near $3 \mathrm{yr}$ has easily escaped attention, showing the power of the PM technique with Kepler data. A wide binary such as this with a lower main-sequence secondary is not expected to have any impact on the formation of the Ap star.

\subsubsection{TIC 77128654, HD 97127}

TIC 77128654 is a known roAp star discovered in SuperWASP data by Holdsworth et al. (2014), who referred to it in a brief form as J1110. They found a pulsation frequency at $106.61 \mathrm{~d}^{-1}$ with an amplitude of $0.66 \mathrm{mmag}$ through the WASP filter. In Fig. 1 we also find a peak at $106.61 \mathrm{~d}^{-1}$ with an amplitude of 0.49 mmag through the redder TESS filter, in good agreement with the WASP result. We also detect a second pulsation frequency at $107.88 \mathrm{~d}^{-1}$ with an amplitude of $60 \pm 9 \mu \mathrm{mag}$. The frequency separation is $15 \mu \mathrm{Hz}$, which is possibly half the large separation. Holdsworth et al. (2014) classified the star as F3p with $T_{\text {eff }}=6500 \mathrm{~K}$, similar to the TIC $T_{\text {eff }}=6700 \mathrm{~K}$ given in Table 1. This is one of the coolest roAp stars.

\subsubsection{TIC 158216369, KIC 7018170}

TIC 158216369 is a known roAp star discovered by Hey et al. (2019) in Kepler long cadence data using superNyquist asteroseismology (Murphy et al. 2013). They found one frequency quintuplet and two triplets equally split by $0.159 \mu \mathrm{Hz}$, which they inferred to be the rotation frequency, giving a rotation period of $P_{\text {rot }}=72.7 \mathrm{~d}$. The amplitudes of all the frequencies they detected are too low for detection in the TESS data, where the noise peaks are around $400 \mu \mathrm{mag}$ as a consequence of the faintness of the $\operatorname{star}(V=13.26)$.

\subsubsection{TIC 158275114, BD+443063}

TIC 158275114 (KIC 8677585) was observed for the full $4 \mathrm{yr}$ of the Kepler main mission in long cadence and for $830 \mathrm{~d}$ in short cadence (1 min). Balona et al. (2013) studied this star in detail, finding frequency variability and an interesting, unexplained low frequency at $3.142 \mathrm{~d}^{-1}$. The TESS data do show several peaks in the frequency range of those found by Balona et al. (2013), but do not add to the much higher frequency resolution, higher signal-to-noise Kepler data.

\subsubsection{TIC 286992225, TYC 2553-480-1}

TIC 286992225 is a known roAp star discovered in SuperWASP data by Holdsworth et al. (2014), who referred to it in a brief form as $\mathrm{J} 1430$. They found a pulsation at $235.5 \mathrm{~d}^{-1}(P=6.1 \mathrm{~min})$ which is at the high frequency end of the roAp star range. The TESS data clearly show two pulsation frequencies (Fig. B.5) at $v_{1}=235.541 \mathrm{~d}^{-1}, A_{1}=545 \mu \mathrm{mag}$ and $v_{2}=232.525 \mathrm{~d}^{-1}, A_{2}=$ $323 \mu \mathrm{mag}$. The separation between them is $35 \mu \mathrm{Hz}$. Using the Gaia EDR3 value of parallax and a bolometric correction of 0.02 gives $L=10.37 L_{\odot}$ and a scaled $\Delta v$ of $66 \mu \mathrm{Hz}$. The separation between the two frequencies of $35 \mu \mathrm{Hz}$ is therefore half the large 
separation. The relative amplitudes suggest that $v_{2}$ may be from a quadrupole mode and $v_{1}$ from a dipole mode. However, roAp stars do not show equipartition of mode energy, hence this is speculative.

\subsection{The $\delta$ Sct stars}

Murphy et al. (2020) showed from models that $\delta$ Sct lowovertone $p$ mode pulsations, and $\gamma$ Dor high-overtone $g$ mode pulsations, are damped in Ap stars with field strengths above about $1 \mathrm{kG}$. They showed a case study of KIC 11296437 , the first known roAp star that shows both high-overtone $p$ modes typical of roAp stars, and low-overtone $p$ modes as in $\delta$ Sct stars. Magnetic Ap stars with $\delta$ Sct pulsations are rare, hence our finding of at least 9 among our 67 ssrAp stars is notable. TIC 127959761 and TIC 331644544 are unusual for $\delta$ Sct stars, and especially for such low-amplitude $\delta$ Sct stars, in being singly periodic. Perhaps that is related to the magnetic nature of these stars. Remarkably, TIC 331644544 is also an unusual eclipsing binary (Sect. 2.3). The $\delta$ Sct frequency range for these stars is illustrated in Fig. 2.

We note in Fig. 2 that there are no obvious $g$ modes excited. That is consistent with Hideyuki Saio's magnetic models (Murphy et al. 2020) that show high order $g$ modes, as in $\gamma$ Dor stars, suppressed in Ap stars. Those same models do allow low overtone $p$ modes to be excited for field strengths less than about $1 \mathrm{kG}$, and the fundamental radial mode to be excited for a field strength up to $4 \mathrm{kG}$. It is further notable that all but one of the nine stars presented have exceedingly low amplitudes - under $0.6 \mathrm{mmag}$. These stars would not have been found in ground-based observations, hence it is unsurprising that we only now are discovering them with TESS data, with their combination of higher precision and longer duration, and more $\delta$ Sct stars being observed. Murphy et al. (2020) pointed out, 'Other roAp stars with low field strengths should also be found with $\delta$ Sct pulsations, similar to KIC 11296437'. As a by-product of our search for ssrAp stars, that appears to be what we have found with these nine (and possibly more) $\delta$ Sct stars.

It is important to determine the magnetic field strengths of all of the ssrAp stars, including these $\delta$ Sct stars. Only TIC 40564267 has a literature measurement (Table 1), and that is $\left\langle B_{z}\right\rangle_{\mathrm{rms}}=0.5 \mathrm{kG}$, consistent with the models of Murphy et al. (2020). We do not know whether any of these Ap $-\delta$ Sct stars are binaries with one Ap and one $\delta$ Sct star. Murphy et al. (2020) showed that was not the case for KIC 11296437. Spectra for the stars shown in Fig. 2 will resolve this, as in such a pair both components should be visible. As those spectra will be obtained for magnetic field measurement, this question will be answered.

\subsection{Eclipsing binaries}

Magnetic Ap (or late Bp) stars in eclipsing binaries are rare. To the best of our knowledge, only five have been reported in the literature: HD 123335 (Bp He wk Sr; Hensberge et al. 2007), HD 66051 (A0p Si; Kochukhov et al. 2018), HD 99458 (Skarka et al. 2019), HD 62658 (B9p Si; Shultz et al. 2019b), and HD 102797 (B9p Si; Paper I). The spectral types are from Renson \& Manfroid (2009), except for HD 99458, whose peculiarity was noted by Skarka et al. (2019). For HD 102797 we cannot definitely rule out the possibility that the eclipses may originate from a contaminating star in the TESS field.
The low rate of occurrence of eclipsing binaries among Ap stars is not very surprising, since the incidence of close binaries with Ap stars is very low, and eclipses are low probability in the longer period Ap binaries. Yet we have found two, long period, eclipsing systems amongst the ssrAp stars. Their light curves are shown in Fig. 3 where only one eclipse is seen in each case, hence the orbital periods are longer than $27 \mathrm{~d}$. In both cases the eclipse depths are 3-4 mmag, giving a ratio of radii of $R_{2} / R_{1} \approx$ 0.06 , assuming that these are transits, and not grazing eclipses. For a roughly estimated radius for the primaries of $R_{1} \approx 2 R_{\odot}$, both of the companions have radii around $R_{2} \approx 0.12 R_{\odot}$; that is they are lower main-sequence companions, which is plausible. We examined the target pixels files for these two stars and find that the eclipse signals for both stars come from the brightest pixels centred on the targets. All Gaia sources within these pixels are at least 5 mag fainter. It is unlikely that the eclipses arise from a background contaminator.

With a rough estimate of the primary mass and radius for both of these binaries to be $2 M_{\odot}$ and $2 R_{\odot}$, respectively, the duration of the transits, which is the time it takes the secondary to cross a distance of $2 R_{\odot}$ assuming a circular orbit, allows an estimate of the orbital velocity of the secondary, which then can be translated into a rough estimate of the semi-major axis of the orbit and the orbital period. While this is crude, it gives approximate orbital periods of $50 \mathrm{~d}$ for TIC 115054217 and $450 \mathrm{~d}$ for TIC 331644544. It is unlikely that the companions of these stars have any impact on the Ap star.

With orbital periods of that order, compliance of these two systems with the conjecture of Mathys (2017) that, barring synchronisation, short rotational and orbital periods are mutually exclusive, would require the rotation periods of the Ap components to be longer than $\sim 50 \mathrm{~d}$. This is not implausible given the apparent lack of rotation-like variability over a TESS sector. The two systems would then lie in the upper left quadrant of Fig. 16 of Mathys (2017).

\section{Discussion}

For 22 of the 67 stars of Table A.1, there exists some independent information about the variability and/or rotation, such as published values of the rotation period and of $v \sin i$, as well as line profile characterisation from either the literature or our own collection of high-resolution spectra.

There are two stars that show sharp lines in high-resolution spectra, hence that have low projected equatorial velocities $v \sin i$, for which variation periods shorter than $27 \mathrm{~d}$ have been reported in the literature: HD 89069 and HD 148330. The $18 \mathrm{~d}$ value of the period of the former that was published by Netopil et al. (2017) can be reconciled with the sharpness of its spectral lines even if neither the inclination $i$ of the rotation axis to the line of sight nor the obliquity $\beta$ of the magnetic axis are exceptionally small. Other similar occurrences are known, such as the prototypical Ap star $\beta$ CrB (=HD 137909), which has clearly resolved magnetically split lines while showing rather large amplitude variations (including a reversing mean longitudinal magnetic field) along its $18.5 \mathrm{~d}$ rotation period (Wolff \& Wolff 1970). While there is a low frequency bump in the amplitude spectrum of HD 89069 (see Fig. B.6), it is not typical of Ap rotation. For HD 148330, the TESS photometry sets a very stringent upper limit of the variability, 3 to 4 orders of magnitude lower than the peak-to-peak amplitude of the data on which the 4.28 value of the period proposed by Ziznovsky \& Romanyuk (1990) is based. This leads to the 

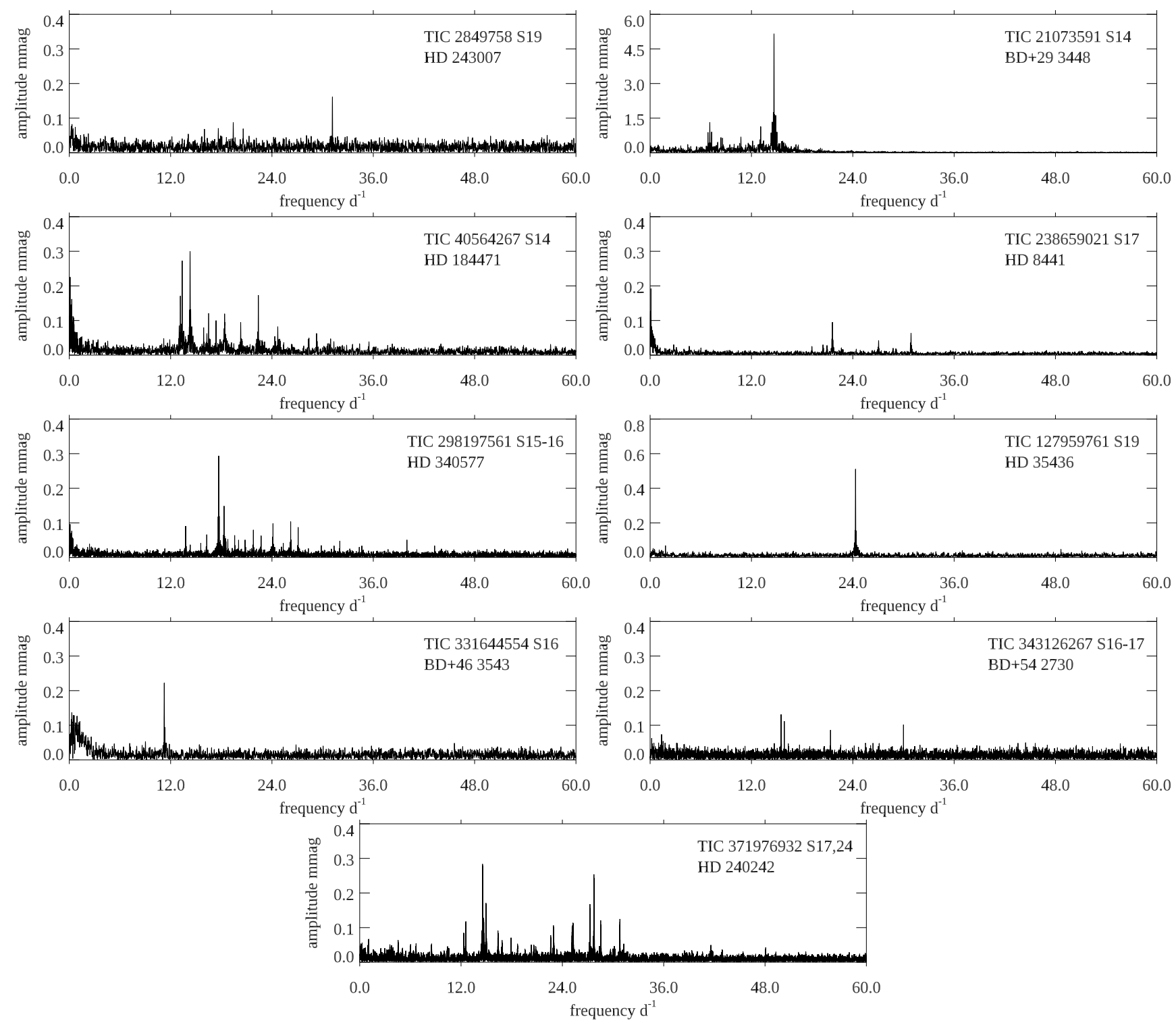

Fig. 2. Amplitude spectra for the nine $\delta$ Sct stars. We note the different ordinate scales for TIC 21073591 and TIC 127959761 . With the exception of TIC 21073591, they are all very low amplitude pulsators, as is predicted for low-overtone $\delta$ Sct pulsation in Ap stars.
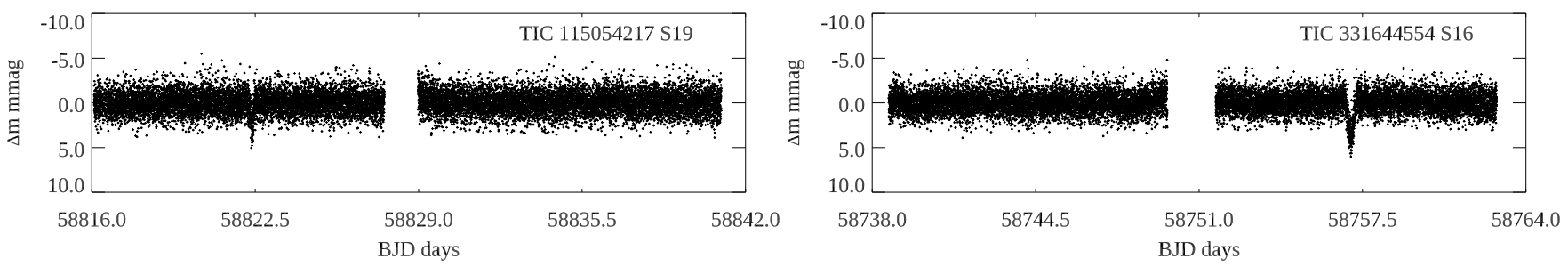

Fig. 3. Light curves of two eclipsing binaries among the ssrAp stars. There is only one eclipse for each star, hence the orbital period is longer than the TESS sector; i.e. longer than $27 \mathrm{~d}$, in these cases.

suspicion that this value is spurious. The lack of photometric variability of the star over a TESS sector and the sharpness of its spectral lines consistently suggest that its rotation period is (very) long.

In contrast with the stars discussed above, we could not find any information about the profiles of the spectral lines of HD 22860. The highest amplitude low-frequency peak in the TESS data is at $v_{\mathrm{rot}}=0.192 \mathrm{~d}^{-1}$. The corresponding value of the rotation period, $P_{\text {rot }}=5.21$, is typical of Ap rotation, but it differs by $1 \mathrm{~d}$ from the published value $\left(P_{\text {rot }}=6.2\right.$ Wraight et al. 2012), of which it is not an alias, and the variation amplitude is very low. This star represents a good illustration of the need for high resolution spectroscopy to complement the present, photometry-based search. Because the periodic variability that is suggested by the low-frequency peaks in the amplitude spectra of the TESS observations (see Fig. B.7) is just above 
the threshold of significance, we cannot confidently decide if HD 22860 has a rotation period of a few days, typical of the majority of the Ap stars, or if it may be rotating very slowly. In the former case, the low photometric amplitude could indicate that either the inclination $i$ or the obliquity $\beta$ is small. With a small $\beta$, the spectral lines should show significant broadening (unless $i$ is also small, but the probability that both $i$ and $\beta$ are small is low).

For the remaining 18 stars of Table A.1 for which complementary information about the periods and/or the line profiles is available, this information is fully compatible with the interpretation that the lack of variability over a TESS sector (or more) reflects the occurrence of (very) slow rotation. As there are plausible explanations for the apparent inconsistencies affecting the three cases discussed above, the present analysis of the northern ecliptic hemisphere TESS observations supports the conclusion drawn from the consideration of the southern ecliptic hemisphere part of the survey (Paper I), that most of the constant stars that are identified with our search technique belong, with a high probability, to the group of the ssrAp stars.

In Paper I, we showed that some stars that were expected to be constant over $27 \mathrm{~d}$ were missed by our search. The degree of incompleteness of the achieved detections could not be fully assessed due to the small number statistics. Eleven of the $33 \mathrm{Ap}$ stars known to have an accurately determined rotation period longer than $50 \mathrm{~d}$ according to the critical compilation of ssrAp stars by Mathys (2020) are located in the northern ecliptic hemisphere. Of these 11 stars, seven were found to be constant over $27 \mathrm{~d}$. The four that were not are HD 5797, HD 158919, HD 188041, and HD 200311. For three of these stars, no 2-min cadence data are available: HD 158919 and HD 188041 fell outside the detector limits, while HD 200311 was only observed in 30-min cadence. Observations of HD 5797 (TIC 256106349) were obtained in three sectors. Three clear frequency peaks are visible. These are possibly caused by $g$ mode pulsations, but their periods and period separations do not obviously fit the $P-\Delta P$ theoretical relations of Ouazzani et al. (2017), and $g$ modes are previously not known, and are not expected in Ap stars, making this star unusual. While these low frequencies led to the rejection of the star in our analysis, there is no indication of variability with the published period, $P_{\text {rot }}=68.05$ (Dukes \& Adelman 2018) in the pipeline reduced data. However, in the data that have not been pipeline reduced (SAP data), there are significant variations that could plausibly be the rotation signal. This case probably represents an example of removal of an astrophysical signal by the pipeline. See also Fig. 4 of Holdsworth et al. (2021) for another example of the occasional interference of the pipeline with the astrophysical signal in TESS observations.

In summary, of the eight known ssrAp stars of the northern ecliptic hemisphere that have been observed in 2-min cadence by TESS, seven were duly detected in our search. The eighth one was missed only because of a reduction artefact. This is consistent with the conclusion reached in Paper I, that while our survey does not return a complete list of the most slowly rotating Ap stars, its incompleteness is not related to the physical properties of the missed stars. Accordingly, its outcome should lend itself to unbiased studies of possible relations between slow rotation and other physical properties of Ap stars.

On the other hand, while for all the southern ecliptic hemisphere candidates identified in Paper I whose rotation periods were accurately known, these periods were all (much) longer than $50 \mathrm{~d}$ (the shortest one was $\sim 155 \mathrm{~d}$ ), three of the candidates identified in the present study have periods between $27 \mathrm{~d}$ and
$49 \mathrm{~d}$, and and four more have periods shorter than $120 \mathrm{~d}$. This illustrates the suitability of our technique also to identify moderately slow rotators.

\section{Magnetic fields}

Figure 4 shows the distribution of the phase-averaged mean magnetic field modulus $B_{0}$ for all of the stars that were identified in Paper I and this paper as not showing photometric variability over a TESS sector, and for which magnetic field measurements have been obtained. As in Paper I, for those stars for which no mean magnetic field modulus determinations are available, an estimate of $B_{0}$ was computed by dividing the phase-averaged value of the mean quadratic field, $Q_{0}$, by 1.28 (the slope of the correlation between these two field moments, according to Mathys 2017); when only mean longitudinal magnetic field measurements had been obtained, we used $B_{0}=3\left\langle B_{z}\right\rangle_{\mathrm{rms}}$, which is actually a lower limit. To reflect this difference, the corresponding parts of the histogram are filled in blue, while those parts based on measured values of $\langle B\rangle$ or $\left\langle B_{\mathrm{q}}\right\rangle$ are filled in red.

Besides the obvious implications of the above-mentioned usage of lower limits of $B_{0}$ for those stars for which $\left\langle B_{z}\right\rangle$ is the only magnetic moment that has been measured, some additional caution is required when drawing conclusions from consideration of Fig. 4 . The correlation between $B_{0}$ and $Q_{0}$ is sufficiently tight (see Fig. 12 of Mathys 2017) that the usage of $Q_{0} / 1.28$ instead of $B_{0}$ in the absence of $\langle B\rangle$ measurements should at most have a moderate impact on the overall distribution. Of more concern is the fact that magnetic measurements have been obtained over at least a full rotation cycle for only 12 of the 42 stars on which the histogram is based. These 12 stars are the only ones for which $B_{0}$ can indeed be regarded as an average over all the phases; for the remaining 30 stars, it is only an average over the existing measurements, with an incomplete and uneven phase coverage. The resulting uncertainty on the actual value of $B_{0}$ is limited for those stars for which measurements of the mean magnetic field modulus or of the mean quadratic magnetic field are available, since the ratio between the extrema of $\langle B\rangle$ has never been observed to exceed 2, and in most cases is lower than 1.3 (see Fig. 9 of Mathys 2017). But the mean longitudinal magnetic field can have very large variation amplitudes, so that the lower limits of the value of $B_{0}$ that are inferred from its consideration should not be over-interpreted. This is why a blue filling of the histogram was used in Fig. 4 to distinguish the 12 stars for which only $\left\langle B_{z}\right\rangle$ measurements have been obtained. However, the fact that, as a group, these 12 stars are concentrated in the lower bins of the histogram (especially the two lowest ones) is consistent with the conjecture that the magnetic field of most of them is too weak to lead to the resolution of magnetically split spectral lines.

Figure 4 represents an augmented version of Fig. 3 of Paper I. Similar to the latter, it shows a lack of stars with $B_{0}>10 \mathrm{kG}$, which is consistent with the conclusion that the strongest magnetic fields $\left(B_{0} \gtrsim 7.5 \mathrm{kG}\right)$ occur only in those Ap stars with periods shorter than $150 \mathrm{~d}$. It is actually noticeable, although inconclusive, that the three stars of Table A.1 in which the strongest magnetic fields have been measured are also the only three stars of this table that are known to have periods shorter than $50 \mathrm{~d}$, hence that are not ssrAp stars in the strictest sense.

On the other hand, while the lowest bins of the histogram $\left(B_{0} \leq 2 \mathrm{kG}\right)$ are relatively more populated than for the southern ecliptic survey alone, the rate of occurrence of long-period candidates still remains less in the $0-3 \mathrm{kG} B_{0}$ range than in the $3-6 \mathrm{kG}$ range. The result that for most of the stars in the lower 


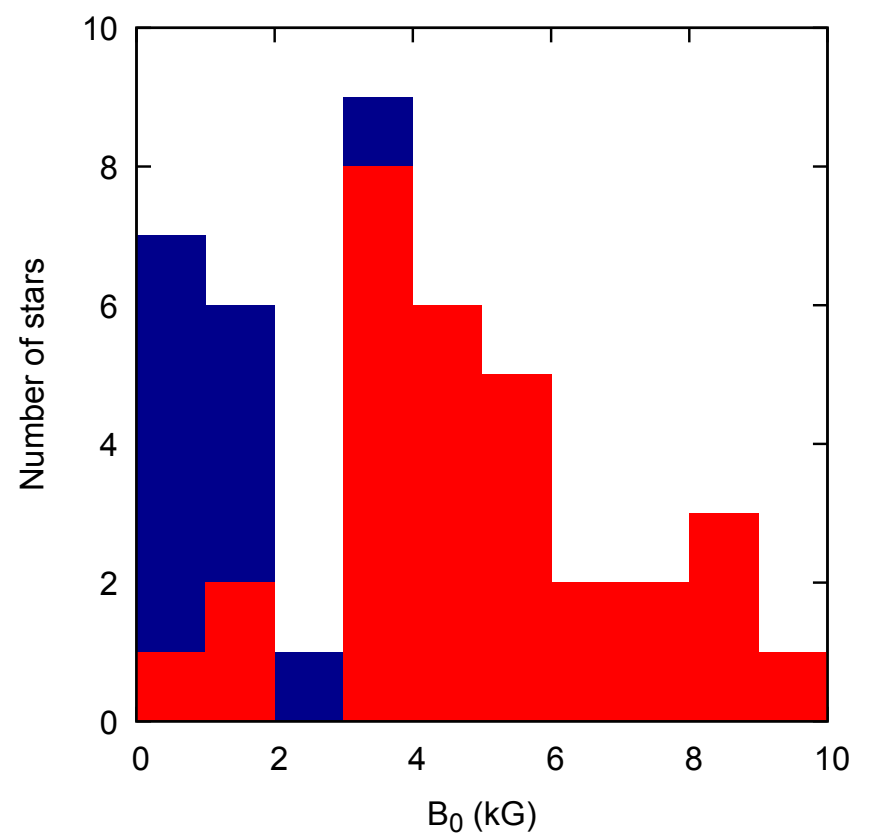

Fig. 4. Distribution of the phase-averaged magnetic field strength $B_{0}$ for the long-period stars of Table A. 1 of this paper and of Table 1 of Paper I. The red part of the histogram corresponds to the stars for which measurements of the mean magnetic field modulus or of the mean quadratic magnetic field are available; for the remaining stars (blue part of the histogram), a lower limit of $B_{0}$ was inferred from the existing mean longitudinal magnetic field measurements.

range, only mean longitudinal magnetic field measurements have been obtained, hence the values of $B_{0}$ used in this histogram are lower limits, only strengthens this result. As the distribution of the root-mean-square longitudinal magnetic fields among all Ap stars sharply peaks at the lowest values (Bychkov et al. 2009), the conclusion that the rate of occurrence of super-slow rotation is considerably lower in weakly magnetic stars than in strongly magnetic ones seems inescapable. This conclusion remains valid even if we take into account six stars of Paper I for which no magnetic measurements have been obtained but whose line profiles (five sharp unresolved ones and one showing only marginal magnetic resolution) indicate that the fields must be considerably lower than $3 \mathrm{kG}$. (There are no such stars in Table A.1).

Admittedly, the mean longitudinal magnetic field measurements compiled in the catalogue of Bychkov et al. (2009) were extracted from a very inhomogeneous collection of sources and they include without distinction $\left\langle B_{z}\right\rangle$ values that are below the threshold of formal significance, so that one may suspect the low field strength population to be overestimated. To assess the extent to which such a bias could affect the above conclusion about the comparatively low rate of occurrence of superslow rotation among weakly magnetic stars, we further compare the present sample of ssrAp candidates with the volume-limited sample of Ap stars studied by Sikora et al. (2019b). We plotted in Fig. 5 the distribution of the dipole field strengths $B_{\mathrm{d}}$ determined by those authors in the same format as the $B_{0}$ distribution that we derived for the ssrAp candidates (Fig. 4). Although the values of the phase-averaged magnetic field strength $B_{0}$ and of the dipole field strength $B_{\mathrm{d}}$ (which cannot be determined for most of the ssrAp candidates) are different from each other, the histograms of Figs. 4 and 5 still lend themselves to meaningful comparison, in the following way.

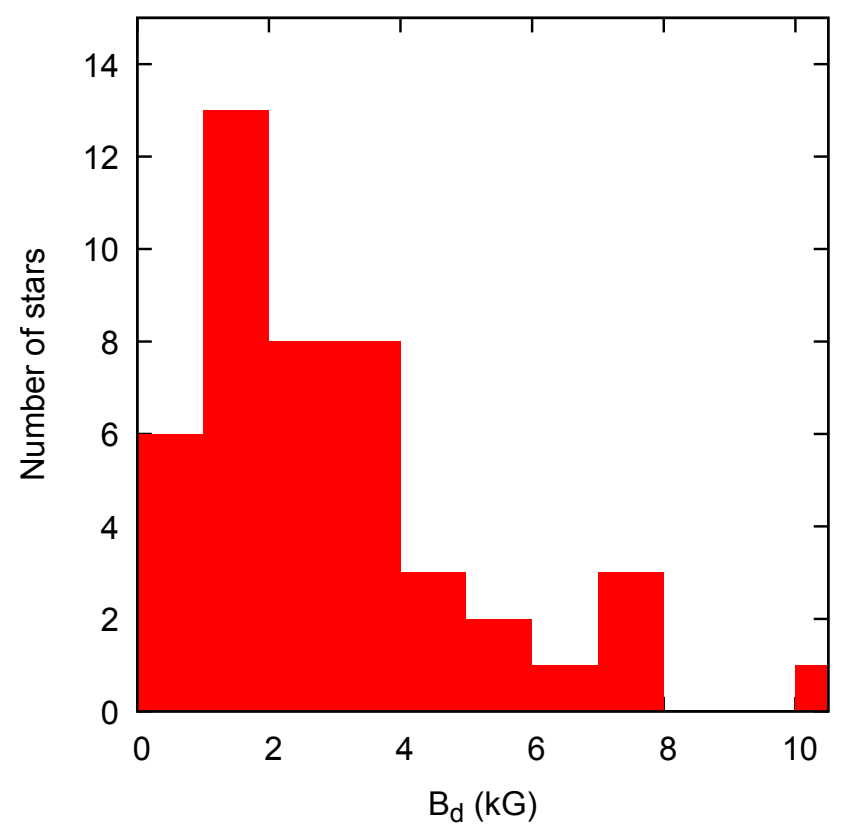

Fig. 5. Distribution of the magnetic dipole field strength $B_{\mathrm{d}}$ for the Ap stars of the volume-limited sample of Sikora et al. (2019b).

The sample of Sikora et al. (2019b) comprises 27 stars in the range $0 \leq B_{\mathrm{d}}<3 \mathrm{kG}$ and 13 stars in the range $3 \leq B_{\mathrm{d}}<6 \mathrm{kG}$. The ssrAp candidates for which magnetic field data exist include 14 stars with $0 \leq B_{0}<3 \mathrm{kG}$ and 20 stars with $3 \leq B_{0}<6 \mathrm{kG}$. One should keep in mind that for most of the stars in the range $[0,3] \mathrm{kG}$, the value of $B_{0}$ is an underestimate of the actual phaseaveraged magnetic field strength, so that based on the latter, several of the stars that appear in the blue parts of the $[0,3] \mathrm{kG}$ interval in the histogram of Fig. 4 may have $B_{0} \geq 3 \mathrm{kG}$.

The ranges of magnetic field values do not correspond to the same physical quantity in the two samples shown in Figs. 4 and 5 , so that the absolute numbers of stars in them are not directly comparable. But the difference between the relative populations of the lower and higher ranges of magnetic field strengths in the two samples can be meaningfully compared. Namely, in the sample of Sikora et al. (2019b), the lower range $([0,3] \mathrm{kG})$ is considerably more populated than the higher one $([3,6] \mathrm{kG})$. This is the opposite for the ssrAp candidates sample, for which the lower range is definitely less populated than the higher one, probably even more so than the plain counts suggest, if one makes allowance for the fact that a number of the stars seemingly in the lower sample likely belong to the higher one. Thus, comparison of our sample with the one of Sikora et al. (2019b) unambiguously confirms the lower rate of occurrence of weakly magnetic stars than of strongly magnetic stars among the ssrAp candidates.

A particularly intriguing feature of the histogram of Fig. 4 is the apparent gap in the $B_{0}$ distribution between 2 and $3 \mathrm{kG}$. Admittedly, the reality of this gap will need to be confirmed by obtaining more precise constraints on the actual value of $B_{0}$ than the uncertain lower limits based on mostly incomplete sets of measurements of the mean longitudinal magnetic field that prevail in the three lowest bins of the histogram. But its coincidence with the apparent bimodality of the mean magnetic field modulus distribution advocated by Mathys (2017) is noteworthy. As already noted by Mathys et al. (1997), Mathys (2017) confirmed that for none of the known Ap stars with resolved magnetically split lines is the average $B_{0}$ of the mean magnetic field modulus 
Table 1. Summary.

\begin{tabular}{lrrr}
\hline \hline Star type & South & North & Total \\
\hline Original sample & 1014 & 492 & 1506 \\
ssrAp candidates & 60 & 67 & 127 \\
Resolved magnetically split lines & 19 & 8 & 27 \\
Sharp unresolved lines & 10 & 7 & 17 \\
$v$ sin $i \lesssim 20 \mathrm{~km} \mathrm{~s}^{-1}$ & 0 & 3 & 3 \\
Broad lines $/ v$ sin $i>20 \mathrm{~km} \mathrm{~s}^{-1}$ & 6 & 0 & 6 \\
& & & \\
Accurate rotation period values & 6 & 14 & 20 \\
$P_{\text {rot }}>50 \mathrm{~d}$ & 6 & 11 & 17 \\
$26 \mathrm{~d}<P_{\text {rot }}<50 \mathrm{~d}$ & 0 & 3 & 3 \\
$P_{\text {rot }}<26 \mathrm{~d}$ ? & 0 & 3 & 3 \\
Stars with magnetic field measurements & 24 & 17 & 41 \\
Definite roAp stars & 12 & 5 & 17 \\
Possible roAp stars & 1 & 8 & 9 \\
Fraction of roAp stars & $22 \%$ & $\leq 19 \%$ & $\leqslant 20 \%$ \\
Definite $\delta$ Sct stars & 2 & 9 & 11 \\
Possible $\delta$ Sct stars & 1 & 5 & 6 \\
Possible eclipsing binaries & 1 & 2 & 3 \\
\hline
\end{tabular}

over a rotation cycle lower than $\sim 2.8 \mathrm{kG}$, although it should be possible to resolve magnetically split lines down to $\langle B\rangle \sim 1.7 \mathrm{kG}$ in most of the spectra analysed in the considered studies. He then argued that there exists a distinct population of Ap stars, separate from that of the Ap stars with resolved magnetically split lines, whose mean magnetic field moduli, averaged over a rotation period, are weaker than $\sim 2 \mathrm{kG}$. This conclusion is based on the consideration of published estimates of the magnetic fields that are essentially comparable to the mean magnetic field modulus or to the mean quadratic magnetic field. The double-peaked histogram shown in Fig. 4 is strikingly consistent with the bimodal distribution suggested by Mathys (2017). Confirming the existence of a gap in the distribution of the magnetic field strengths of the ssrAp stars between $\sim 2 \mathrm{kG}$ and $\sim 3 \mathrm{kG}$ is one of the important objectives of the systematic study of the magnetic fields of the candidate ssrAp stars that we intend to carry out.

\section{Conclusion}

Table 1 summarises the results of the search for Ap stars that show no low-frequency variability over a TESS sector in the southern ecliptic hemisphere (Paper I) and in the northern ecliptic hemisphere (this work). A total of 127 such stars were identified, split almost evenly between the two hemispheres. Spectroscopic information is available for 53 of them $(\sim 2 / 3$ of which are in the southern ecliptic hemisphere), either in the literature or in our own collection of spectra. Of these 53, 27 have spectral lines resolved into their magnetically split components, and 17 show sharp spectral lines that are unresolved at high resolving powers $\left(5 \times 10^{4} \lesssim R \lesssim 10^{5}\right)$. Almost all of these 44 $(=27+17)$ stars likely are genuine slow rotators. Three more stars plausibly belong to this group, as their line profiles do not show significant rotational broadening in medium resolution spectra, indicating values of $v \sin i \lesssim 20 \mathrm{~km} \mathrm{~s}^{-1}$. The remaining six stars for which we have spectroscopic information (all from the southern ecliptic hemisphere) have broad or very broad spectral lines. If the magnetic obliquities of Ap stars are randomly distributed, the interpretation that we proposed in Paper I - that these six stars are fast-rotating stars in which the angle between the mag- netic and rotation axes is small - is consistent with their rate of occurrence, six out of 1506 stars in the entire sample that was analysed, or $0.40 \%$.

Values of the rotation period have been derived for four of the stars for which we have no line profile information (one from Paper I). Three of them exceed $70 \mathrm{~d}$; the published period of the fourth star, $P_{\text {rot }}=6.27$, is not supported by the TESS observations.

In summary, the available line profile and period information is consistent with the view that Ap stars that show no variability over a TESS sector are (very) slow rotators for 50 of the 57 stars for which such information was found. The few exceptions can be reasonably justified, mostly in terms of low magnetic obliquity. This result, based on an increased sample, strengthens the conclusion from Paper I that the search technique that we applied is effective and reliable. Accordingly, we expect almost all the stars of Table A.1 for which neither line profile nor period constraints have been published yet to be genuine long-period stars. In the present study, we identify in this way 46 new ssrAp star candidates. Together with the 23 candidates from Paper I, they open the prospect of increasing by a factor of almost three the number of known ssrAp stars, in a mostly bias-free way. In particular, with the technique discussed here, the identification of ssrAp stars is fully independent of their magnetic fields, which makes it possible to study the existence of possible dependences between the magnetic field strengths and the rotation periods.

The next, critical step in this study is to obtain high-resolution spectra of the 69 ssrAp star candidates, to confirm that they have low projected equatorial velocities $v \sin i$ and to obtain preliminary estimates of their magnetic fields. Once this is done, multiepoch spectroscopic or spectropolarimetric observations of the confirmed candidates will have to be obtained so as to constrain their rotation periods and characterise their magnetic variations, either from consideration of their mean magnetic field moduli or of their mean longitudinal magnetic fields (or both).

Despite the incompleteness of the rotation and magnetic information that has been acquired until now, the present study strengthens the tentative conclusion reached in Paper I that the rate of occurrence of super-slow rotation appears significantly lower in weakly magnetic Ap stars than in those that are strongly magnetic. One of the main objectives of follow-up studies will be to characterise the low end of the magnetic field strength distribution among ssrAp stars. This is an area of the parameter space that has remained almost unexplored until recently. It will be of particular interest to test the possible existence of a gap between $\sim 2 \mathrm{kG}$ and $\sim 3 \mathrm{kG}$ in which no ssrAp star is found. The existence of such a gap, which was first proposed by Mathys (2017) as part of a general study of the Ap stars with resolved magnetically split lines, and which appears further supported by the results of our present search for ssrAp stars, would potentially have profound implications for the understanding of the origin of super-slow rotation in Ap stars. Indeed, it would suggest the existence of two distinct populations, consisting of weakly and strongly magnetic Ap stars, respectively, whose rotational evolution would follow different paths and involve different physical mechanisms.

Another result from Paper I that is further confirmed by the present study is the high rate of occurrence of roAp stars among ssrAp candidates. A definitive quantitative characterisation of this rate cannot be given yet: Table A.1 includes five definite roAp stars and eight candidates that have not been fully confirmed yet. We expect that most of them will eventually be, but even being conservative, the 17 definite roAp stars summed over the two hemispheres represent $14 \%$ of the 120 long-period Ap 


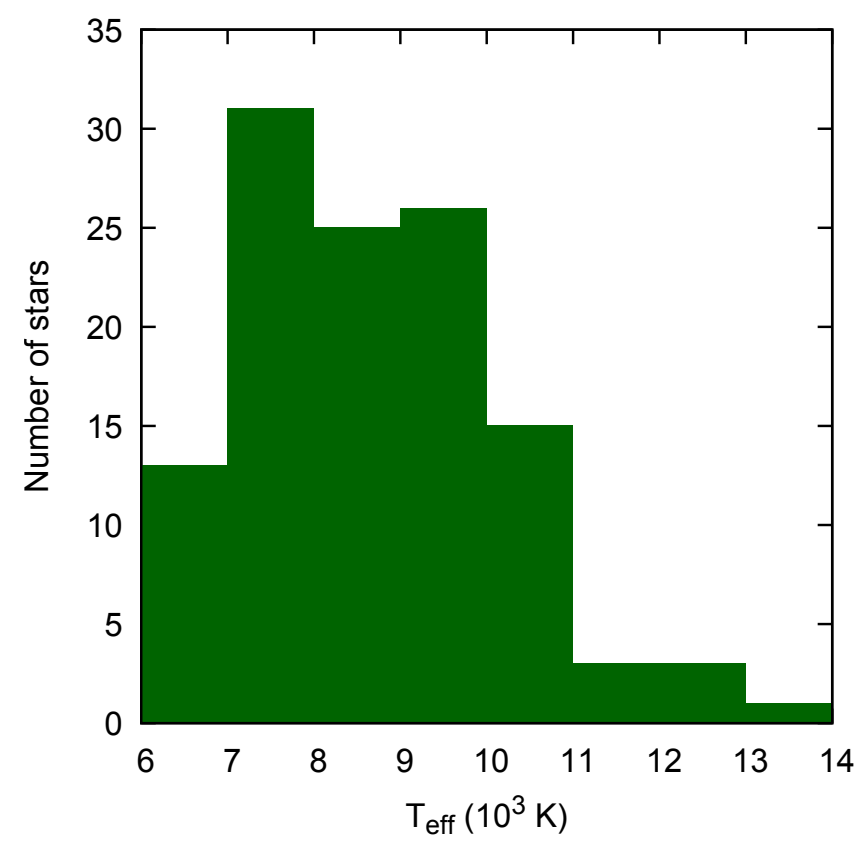

Fig. 6. Distribution of the effective temperatures of the ssrAp candidates from Table A.1 and from Table 1 of Paper I.

stars from the combined lists of Paper I and of the present study. Even this lower limit is considerably higher than the fraction of roAp stars reported for the $>1000$ Ap stars observed by TESS in Sectors 1-13 (Cunha et al. 2019; Holdsworth et al. 2021). The incidence of the roAp phenomenon is low among Ap stars in general, but definitely higher for ssrAp stars.

One might possibly suspect that this difference is not directly related to slow rotation itself, but to some other distinctive property of the ssrAp stars as a group. One obvious candidate for such a connection is the distribution of the effective temperatures. From consideration of the roAp star lists of Smalley et al. (2015) and Holdsworth et al. (2021) (which partly overlap with each other), one can see that these stars are found in a narrow $T_{\text {eff }}$ range, 6000-9000 K, with a distribution sharply peaked towards the centre of this interval. This contrasts with the $T_{\text {eff }}$ distribution of the ssrAp candidates identified in Paper I and in the present study, as shown in Fig. 6. This distribution is much broader and shallower, with $94 \%$ of the stars in the $T_{\text {eff }}$ range $6000-11000 \mathrm{~K}$. It is almost flat from 7000 to $10000 \mathrm{~K}$, an interval that accounts for $70 \%$ of the ssrAp candidates. In summary, the ssrAp candidates span a much wider range of temperatures than the roAp stars, in a more uniform way, so that the comparatively high rate of occurrence of roAp stars among ssrAp stars does not appear to be primarily temperature related. What causes it remains to be determined.

Also surprising on the asteroseismology front is the discovery that nine of the 66 long-period Ap stars from Table A.1 are definite $\delta$ Sct stars, and five more are candidate $\delta$ Sct stars. This is not only unexpected, but also in marked contrast with the southern ecliptic hemisphere statistics. Indeed, there were only two definite $\delta$ Sct stars and one candidate in Table 1 of Paper I.

These findings raise intriguing questions about the connections between rotation, pulsation and magnetic field in Ap stars. They represent an additional incentive to pursue systematic spectroscopic studies of the candidate ssrAp stars identified here and in Paper I, with a view to constraining their rotational and magnetic properties, as well as to exploit the next TESS data releases to further increase the number of ssrAp star candidates.
Acknowledgements. This research has made use of the SIMBAD database, operated at CDS, Strasbourg, France. This paper includes data collected by the TESS mission, which are publicly available from the Mikulski Archive for Space Telescopes (MAST). Funding for the TESS mission is provided by NASA's Science Mission directorate. Funding for the TESS Asteroseismic Science Operations Centre is provided by the Danish National Research Foundation (Grant agreement no.: DNRF106), ESA PRODEX (PEA 4000119301) and Stellar Astrophysics Centre (SAC) at Aarhus University. We thank the TESS team and staff and TASC/TASOC for their support of this work. We also thank the referee, Gregg Wade, for helpful comments that led to improvements in this paper.

\section{References}

Adelman, S. J. 1981, A\&AS, 44, 265

Aurière, M., Wade, G. A., Silvester, J., et al. 2007, A\&A, 475, 1053

Babcock, H. W. 1958, ApJS, 3, 141

Balona, L. A., Cunha, M. S., Kurtz, D. W., et al. 2011, MNRAS, 410, 517

Balona, L. A., Catanzaro, G., Crause, L., et al. 2013, MNRAS, 432, 2808

Bohlender, D. A., Landstreet, J. D., \& Thompson, I. B. 1993, A\&A, 269, 355

Bychkov, V. D., Bychkova, L. V., \& Madej, J. 2009, MNRAS, 394, 1338

Cunha, M. S., Antoci, V., Holdsworth, D. L., et al. 2019, MNRAS, 487, 3523

Dukes, R. J., Jr., \& Adelman, S. J. 2018, PASP, 130, 044202

Grunhut, J. H., Wade, G. A., Neiner, C., et al. 2017, MNRAS, 465, 2432

Hartkopf, W. I., \& McAlister, H. A. 1984, PASP, 96, 105

Hensberge, H., Nitschelm, C., Olsen, E. H., et al. 2007, MNRAS, 379, 349

Hey, D. R., Holdsworth, D. L., Bedding, T. R., et al. 2019, MNRAS, 488, 18

Holdsworth, D. L., Smalley, B., Gillon, M., et al. 2014, MNRAS, 439, 2078

Holdsworth, D. L., Cunha, M. S., Kurtz, D. W., et al. 2021, MNRAS, 506, 1073

Horch, E. P., van Belle, G. T., Davidson, J. W., Jr., et al. 2015, AJ, 150, 151

Hubrig, S., North, P., \& Schöller, M. 2007, Astron. Nachr., 328, 475

Hümmerich, S., Paunzen, E., \& Bernhard, K. 2016, AJ, 152, 104

Jenkins, J. M., Twicken, J. D., McCauliff, S., et al. 2016, Proc. SPIE, 9913, 99133E

Kochukhov, O., \& Bagnulo, S. 2006, A\&A, 450, 763

Kochukhov, O., Johnston, C., Alecian, E., \& Wade, G. A. 2018, MNRAS, 478, 1749

Kudryavtsev, D. O., \& Romanyuk, I. I. 2012, Astron. Nachr., 333, 41

Mathys, G. 2017, A\&A, 601, A14

Mathys, G. 2020, in Stellar Magnetism: A Workshop in Honour of the Career and Contributions of John D. Landstreet, eds. G. Wade, E. Alecian, D. Bohlender, \& A. Sigut, Proc. Polish Astron. Soc., 11, 35

Mathys, G., Hubrig, S., Landstreet, J. D., Lanz, T., \& Manfroid, J. 1997, A\&AS, 123,353

Mathys, G., Romanyuk, I. I., Kudryavtsev, D. O., et al. 2016, A\&A, 586, A85

Mathys, G., Kurtz, D. W., \& Holdsworth, D. L. 2020, A\&A, 639, A31

Murphy, S. J., Shibahashi, H., \& Kurtz, D. W. 2013, MNRAS, 430, 2986

Murphy, S. J., Moe, M., Kurtz, D. W., et al. 2018, MNRAS, 474, 4322

Murphy, S. J., Saio, H., Takada-Hidai, M., et al. 2020, MNRAS, 498, 4272

Netopil, M., Paunzen, E., Hümmerich, S., \& Bernhard, K. 2017, MNRAS, 468, 2745

Ouazzani, R.-M., Salmon, S. J. A. J., Antoci, V., et al. 2017, MNRAS, 465, 2294

Preston, G. W. 1971, ApJ, 164, 309

Pyper, D. M., \& Adelman, S. J. 2017, PASP, 129, 104203

Renson, P., \& Manfroid, J. 2009, A\&A, 498, 961

Romanyuk, I. I., Semenko, E. A., Kudryavtsev, D. O., Moiseeva, A. V., \& Yakunin, I. A. 2017, Astrophys. Bull., 72, 391

Romanyuk, I. I., Semenko, E. A., Moiseeva, A. V., Kudryavtsev, D. O., \& Yakunin, I. A. 2018, Astrophys. Bull., 73, 178

Schöller, M., Correia, S., Hubrig, S., \& Kurtz, D. W. 2012, A\&A, 545, A38

Schöller, M., Hubrig, S., Fossati, L., et al. 2017, A\&A, 599, A66

Shultz, M. E., Wade, G. A., Rivinius, T., et al. 2018, MNRAS, 475, 5144

Shultz, M. E., Wade, G. A., Rivinius, T., et al. 2019a, MNRAS, 490, 274

Shultz, M. E., Johnston, C., Labadie-Bartz, J., et al. 2019b, MNRAS, 490, 4154

Sikora, J., Wade, G. A., Power, J., \& Neiner, C. 2019a, MNRAS, 483, 2300

Sikora, J., Wade, G. A., Power, J., \& Neiner, C. 2019b, MNRAS, 483, 3127

Skarka, M., Kabáth, P., Paunzen, E., et al. 2019, MNRAS, 487, 4230

Smalley, B., Niemczura, E., Murphy, S. J., et al. 2015, MNRAS, 452, 3334

Wade, G. A., Kudryavtsev, D., Romanyuk, I. I., Landstreet, J. D., \& Mathys, G. 2000, A\&A, 355, 1080

Wolff, S. C., \& Wolff, R. J. 1970, ApJ, 160, 1049

Wraight, K. T., Fossati, L., Netopil, M., et al. 2012, MNRAS, 420, 757

Zacharias, N., Monet, D. G., Levine, S. E., et al. 2004, Am. Astron. Soc. Meet. Abstr., 205, 48.15

Ziznovsky, J., \& Romanyuk, I. I. 1990, Bull. Astron. Inst. Czechoslov., 41, 118

Zorec, J., \& Royer, F. 2012, A\&A, 537, A120 


\section{Appendix A: List of long rotation period Ap star candidates}

Table A.1 lists the 67 long rotation Ap star candidates that were identified as described in Sect. 2. It is ordered by the TIC numbers, which are given in Column 1. Column 2 gives an alternate identifier: HD number as first choice, then DM number, for stars having these; other identifiers otherwise. The spectral types listed in Column 3 are from the Catalogue of Ap, $\mathrm{HgMn}$ and Am stars (Renson \& Manfroid 2009). For those stars that are not in this catalogue, the spectral type entry was left blank. As mentioned in Sect. 2, these stars were kept in the list only on the basis of more recently published compelling evidence of their Ap nature. The $V$ magnitudes in Column 4 were extracted from the SIMBAD database, while the $T_{\text {eff }}$ and $\log g$ values in Columns 5 and 6 were taken from the TIC. Column 7 identifies the known or suspected roAp stars; unconfirmed cases are flagged with a question mark. Column 8 gives information about the measured magnetic field strengths. All three values correspond to the mean over a rotation period (if known) or over the existing observations (otherwise) of the range spanned by each of the following magnetic field moments: $\left\langle B_{z}\right\rangle_{\mathrm{rms}}$ is the rootmean-square longitudinal field, as defined by Bohlender et al. (1993). It is essentially the quadratic mean of the mean longitudinal magnetic field (the line-intensity weighted average over the stellar disc of the component of the magnetic vector along the line of sight). We denote by $B_{0}$ the average value over a rotation cycle of the mean magnetic field modulus $\langle B\rangle$ (the line-intensity weighted average over the stellar disc of the modulus of the magnetic vector); this is the same quantity as listed in Column 3 of Table 13 of Mathys (2017). The notation $Q_{0}$ refers to the average value over a rotation cycle of the mean quadratic magnetic field $\left\langle B_{\mathrm{q}}\right\rangle$; this is the same quantity as listed in Column 10 of Table 13 of Mathys (2017). The values indicated in Column 8 are based on the references specified in Column 9. Column 10 gives the published values of the rotation periods, from the references appearing in the following column. Column 12 gives an indication of the width or the magnetic resolution of the spectral lines (as a letter) and the $v \sin i$ value (as a number). The resolution information and $v \sin i$ values are extracted from the references indicated in Column 13. The width information for unresolved lines is based on their appearance in high-resolution spectra from our collection (Reference 3). These spectra were obtained at the Observatoire de Haute-Provence with the AURELIE spectrograph fed by the 1.52-m telescope (see Mathys et al. 1997, for details). Column 14, the penultimate column, contains various notes, in particular about variability due to oscillations and binarity. The final column indicates in which TESS 27-d sector(s) the star was observed.

Table A.1. List of long-period Ap stars found by our technique in the TESS Sectors 14-26 data, obtained in the second year of mission operations and covering the northern ecliptic hemisphere.

\begin{tabular}{|c|c|c|c|c|c|c|c|c|c|c|c|c|c|c|}
\hline TIC & HD/Other id & $\begin{array}{l}\text { Spectral } \\
\text { type }\end{array}$ & $\begin{array}{c}V \\
(\mathrm{mag})\end{array}$ & $\begin{array}{l}T_{\text {eff }} \\
(\mathrm{K}) \\
\end{array}$ & $\begin{array}{r}\log g \\
\left(\mathrm{~cm} \mathrm{~s}^{-2}\right) \\
\end{array}$ & roAp & $\begin{array}{c}\left\langle B_{z}\right\rangle_{\mathrm{rms}} / B_{0} / Q_{0} \\
(\mathrm{kG})\end{array}$ & Refs & $\begin{array}{r}P_{\text {rot }} \\
(\mathrm{d}) \\
\end{array}$ & Refs & $\begin{array}{c}\text { Lines }^{(a)} \\
v \sin i \\
\left(\mathrm{~km} \mathrm{~s}^{-1}\right)\end{array}$ & Refs & Notes & $\begin{array}{l}\text { TESS } \\
\text { sectors }\end{array}$ \\
\hline 2689133 & HD 242800 & A0p Si & 10.55 & 8500 & 4.0 & & & & & & & & & S19 \\
\hline 2849758 & HD 243007 & $\mathrm{~A} 1 \mathrm{p} \mathrm{Si}$ & 10.2 & 9000 & 3.6 & & & & & & & & $\delta$ Sct star & S19 \\
\hline 21073591 & $B D+293448$ & A9p Sr & 9.54 & 7600 & 3.6 & & & & & & & & $\delta$ Sct star & S14 \\
\hline 26749633 & KIC 11031749 & & 12.50 & 7000 & 3.9 & roAp & & & & & & & & S14,15 \\
\hline 29715050 & TYC 2653-2787-1 & $\mathrm{A} 0 \mathrm{p} \mathrm{HgSi}$ & 9.08 & 10450 & 4.2 & & & & & & & & & S14 \\
\hline 40564267 & HD 184471 & A9p SrCrEu & 9.00 & 7750 & 3.6 & & $0.5 /-/-$ & 4 & 50.8 & 4 & s & 3 & $\delta$ Sct star & S14 \\
\hline 44488067 & HD 2453 & $\mathrm{~A} 1 \mathrm{p} \mathrm{SrEuCr}$ & 6.91 & 8900 & 3.8 & & $0.8 / 3.7 / 4.3$ & 1 & 521 & 12 & $\mathrm{r}$ & 1 & & S17 \\
\hline 76379777 & HD 247591 & $\mathrm{~A} 1 \mathrm{p} \mathrm{SiCrSr}$ & 10.47 & 9900 & 3.8 & & & & & & & & & S19 \\
\hline 77038207 & HD 96003 & $\mathrm{~A} 3 \mathrm{p} \mathrm{SrCr}$ & 6.87 & 9500 & 3.9 & & $0.2 /-/-$ & 5,6 & & & $<20$ & 5 & & $\mathrm{~S} 22$ \\
\hline 77128654 & HD 97127 & & 9.43 & 6700 & 4.0 & roAp & & & & & & & & S22 \\
\hline 78784187 & HD 249401 & $\mathrm{~A} 2 \mathrm{p} \mathrm{Si}$ & 10.83 & 8150 & 3.6 & & & & & & & & $\delta$ Sct $/ \gamma$ Dor star? & S19 \\
\hline 115054217 & HD 244545 & $\mathrm{~A} 1 \mathrm{p} \mathrm{Si}$ & 10.37 & 8350 & & roAp? & & & & & & & $\mathrm{EB}$ & S19 \\
\hline 115201166 & HD 244640 & A0p SiSr & 9.94 & 9650 & 4.0 & roAp? & & & & & & & & S19 \\
\hline 115820611 & HD 245416 & $\mathrm{~A} 2 \mathrm{p} \mathrm{CrSi}$ & 9.71 & 8150 & 3.7 & & & & & & & & & S19 \\
\hline 116144208 & HD 245726 & Alp SiSr & 10.8 & 8750 & 3.6 & & & & & & & & & S19 \\
\hline 116995376 & TYC 2413-476-1 & $\mathrm{A} 0 \mathrm{p} \mathrm{Sr}$ & 10.82 & 9050 & 3.8 & & & & & & & & $\delta$ Sct star? & S19 \\
\hline 127755719 & HD 243321 & $\mathrm{~A} 0 \mathrm{p} \mathrm{Si}$ & 9.61 & 10000 & 3.9 & & & & & & & & & S19 \\
\hline 127755856 & $\mathrm{BD}+32976$ & $\mathrm{~A} 2 \mathrm{p} \mathrm{Sr}$ & 10.28 & 7600 & 4.2 & roAp? & & & & & & & & S19 \\
\hline 127959761 & HD 35436 & Alp SiSr & 9.55 & 8000 & 3.5 & & & & & & & & $\delta$ Sct star & S19 \\
\hline 154786038 & HD 96571 & $\mathrm{~A} 2 \mathrm{p} \mathrm{SrEu}$ & 7.31 & 8100 & 3.6 & & & & & & & & $\delta$ Sct star? & $\mathrm{S} 14,19,20,26$ \\
\hline 155667362 & HD 276625 & $\mathrm{~A} 7 \mathrm{p} \mathrm{CrEu}$ & 9.94 & 7100 & 3.9 & & & & & & & & & S19 \\
\hline 158216369 & KIC 7018170 & & $13.28^{(b)}$ & 6950 & 4.0 & roAp & & & 72.7 & 13 & & & & S14 \\
\hline 158275114 & $\mathrm{BD}+443063$ & A5p EuCr & 10.19 & 7450 & 4.0 & roAp & $-/ 3.2 /-$ & 7 & & & $\mathrm{r} ; 4.2$ & 2 & & $\mathrm{~S} 14,26$ \\
\hline 163801263 & HD 203922 & $\mathrm{~A} 2 \mathrm{p} \mathrm{SrCrEu}$ & 8.50 & 7600 & 3.6 & & & & & & & & & S15 \\
\hline 165327084 & HD 110066 & $\mathrm{~A} 1 \mathrm{p} \mathrm{SrCrEu}$ & 6.385 & 9000 & 3.9 & & $0.1 / 4.1 /-$ & 1 & $4900 ?$ & 14 & $\mathrm{r}$ & 1 & & S22 \\
\hline 165446000 & $\mathrm{BD}+394435$ & F0p SiSrCr & 9.3 & 7700 & & & & & & & & & & S15 \\
\hline
\end{tabular}

Notes. ${ }^{(a)} \mathrm{r}=$ resolved; $\mathrm{s}=$ sharp; $(\mathrm{s})=$ rather sharp. ${ }^{(b)} V$ magnitude from Zacharias et al. $(2004) .{ }^{(c)}$ TESS photometry definitely does not show any low-frequency variability; the published period may be spurious. ${ }^{(d)} \mathrm{SB} 2$ system; HD 174017 (secondary) is the Ap component (A0p SrCrEu); HD 174016 is a G6III star (Renson \& Manfroid 2009), unresolved by speckle interferometry (Hartkopf \& McAlister 1984; Horch et al. 2015). ${ }^{(e)}$ TESS photometry shows variations with a period of 5.21 , which is typical of rotation, but the amplitude is very low and the period is not an alias of the published value. 
Table A.1. continued.

\begin{tabular}{|c|c|c|c|c|c|c|c|c|c|c|c|c|c|c|}
\hline TIC & HD/Other id & $\begin{array}{l}\text { Spectral } \\
\text { type }\end{array}$ & $\begin{array}{c}V \\
(\mathrm{mag})\end{array}$ & $\begin{array}{l}T_{\text {eff }} \\
(\mathrm{K}) \\
\end{array}$ & $\begin{array}{r}\log g \\
\left(\mathrm{~cm} \mathrm{~s}^{-2}\right) \\
\end{array}$ & roAp & $\begin{array}{c}\left\langle B_{z}\right\rangle_{\mathrm{rms}} / B_{0} / Q_{0} \\
(\mathrm{kG})\end{array}$ & Refs & $\begin{array}{r}P_{\text {rot }} \\
(\mathrm{d}) \\
\end{array}$ & Refs & $\begin{array}{c}\operatorname{Lines}^{(a)} \\
v \sin i \\
\left(\mathrm{~km} \mathrm{~s}^{-1}\right)\end{array}$ & Refs & Notes & $\begin{array}{l}\text { TESS } \\
\text { sectors }\end{array}$ \\
\hline 176902576 & HD 222416 & A0p SiSr & 7.73 & 9850 & 3.9 & & & & & & & & & S17 \\
\hline 185716313 & HD 191742 & $\mathrm{~A} 5 \mathrm{p} \mathrm{SrCr}$ & 8.16 & 8400 & 3.6 & & $0.6 /-/ 1.8$ & 8,9 & & & $\mathrm{~s} ; 6$ & 3,9 & $\delta$ Sct star? & $\mathrm{S} 14,15$ \\
\hline 198781718 & $\mathrm{BD}+293427$ & $\mathrm{~A} 6 \mathrm{p} \mathrm{CrSr}$ & 9.66 & 8000 & 4.2 & & & & & & & & & S14 \\
\hline 202899762 & $\mathrm{BD}+46570$ & $\mathrm{Ap} \mathrm{SrEuCr}$ & 9.63 & 7350 & 3.6 & & & & & & & & & S18 \\
\hline 207468665 & HD 148330 & A2p SiSr & 5.734 & 9700 & 3.8 & & $0.3 /-/-$ & 10 & $4.288^{(c)}$ & 10 & (s); 11.6 & 3,10 & & S16,19,23,25 \\
\hline 233539061 & HD 174016 & & 7.45 & 6225 & & & & & & & & & & All but $\mathrm{S} 18$ \\
\hline 238659021 & HD 8441 & $\mathrm{~A} 2 \mathrm{p} \mathrm{Sr}$ & 6.676 & 9200 & & & $0.1 /-/-$ & 11 & 69.51 & 15 & s & 3 & $\delta$ Sct star & S17 \\
\hline 239801694 & HD 247628 & A5p SiSr & 10.59 & 9050 & 4.1 & & & & & & & & & S19 \\
\hline 251282995 & HD 18078 & $\mathrm{~A} 0 \mathrm{p} \mathrm{SrCr}$ & 8.27 & 8500 & 3.4 & roAp? & $0.7 / 3.5 /-$ & & 1358 & 16 & $\mathrm{r}$ & 1 & & S18 \\
\hline 251976407 & HD 221568 & Alp SrCrEu & 7.55 & 9500 & 3.9 & & $0.7 /-/-$ & 6 & 159.1 & 15 & $\mathrm{~s}$ & 3 & & $\mathrm{~S} 17,24$ \\
\hline 274242788 & HD 92728 & $\mathrm{~A} 0 \mathrm{p} \mathrm{Si}$ & 5.782 & 10050 & 3.9 & & & & & & 22 & 21 & & $\mathrm{~S} 21$ \\
\hline 286462756 & HD 335238 & $\mathrm{~A} 1 \mathrm{p} \mathrm{CrEu}$ & 9.26 & 9350 & 4.2 & & $1.4 / 9.3 / 11.9$ & 1 & 48.7 & 1 & $\mathrm{r}$ & 1 & & S15 \\
\hline 286965228 & HD 127304 & B9p Si? & 6.05 & 9950 & 4.1 & & & & & & & & & S23 \\
\hline 286992225 & TYC 2553-480-1 & $\mathrm{A} 2 \mathrm{p} \mathrm{SrEu}$ & 11.56 & 7500 & 4.3 & roAp & & & & & & & & $\mathrm{S} 23$ \\
\hline 289548946 & $\mathrm{BD}+473253$ & $\mathrm{~A} 0 \mathrm{p} \mathrm{Si}$ & 9.55 & 8800 & 4.0 & & & & & & & & & S15,16 \\
\hline 298197561 & HD 340577 & $\mathrm{~A} 3 \mathrm{p} \mathrm{SrCrEu}$ & 9.09 & 8450 & 3.5 & & & & 116.7 & 17 & & & $\delta$ Sct star & $\mathrm{S} 14,15$ \\
\hline 301918605 & HD 17330 & B7p Si & 7.11 & 10250 & & & $0.6 /-/-$ & 5,6 & & & 23 & 6 & & S18 \\
\hline 301946105 & HD 7410 & $\mathrm{~A} 7 \mathrm{p} \mathrm{CrEu}$ & 9.07 & 7800 & 3.5 & & & & & & & & & S17 \\
\hline 312221714 & HD 248727 & A0p $\mathrm{MnSiCr}$ & 10.34 & 9800 & 3.9 & & & & & & & & $\delta$ Sct star? & S19 \\
\hline 327293700 & HD 9996 & B9p CrEuSi & 6.38 & 10500 & 4.0 & & $0.7 / 4.7 /-$ & 1 & 7850 & 15 & $\mathrm{r}$ & 1 & & $\mathrm{~S} 18$ \\
\hline 331644554 & $\mathrm{BD}+463543$ & $\mathrm{~A} 2 \mathrm{p} \mathrm{Si}$ & 9.75 & 8450 & 4.3 & & & & & & & & $\delta$ Sct star; EB & S16 \\
\hline 341616734 & HD 89069 & A0p SrCrEu & 8.42 & 9550 & 4.0 & & $0.5 /-1-$ & 5 & 19 & 18 & $\mathrm{~s} ;<20$ & 3,5 & & S14,20,21,26 \\
\hline 343126267 & $\mathrm{BD}+542730$ & F0p SiSr & 10.46 & 8000 & 4.2 & & & & & & & & $\delta$ Sct star & S16,17 \\
\hline 346595127 & HD 22860 & B9p Si & 6.86 & 10700 & 4.1 & & & & $6.27^{(e)}$ & 19 & & & & S 18 \\
\hline 347202840 & HD 236298 & $\mathrm{~A} 0 \mathrm{p} \mathrm{Si}$ & 9.45 & 10700 & 4.2 & & & & & & & & & S17,24 \\
\hline 352787151 & BD+35 5094 & F0p SrEu & 9.08 & 6900 & 3.5 & roAp? & & & & & & & & S17 \\
\hline 353371885 & HD 249931 & $\mathrm{~A} 0 \mathrm{p} \mathrm{SiSr}$ & 10.62 & 9100 & 4.3 & & & & & & & & & S19 \\
\hline 368073692 & HD 278204 & B5p Si & 10.45 & 10850 & 4.1 & & & & & & & & & S19 \\
\hline 371976932 & HD 240242 & $\mathrm{~A} 4 \mathrm{p} \mathrm{Si}$ & 10.15 & & & & & & & & & & $\delta$ Sct star & $S 17,24$ \\
\hline 373378410 & $\mathrm{BD}+354488$ & $\mathrm{~A} 2 \mathrm{p} \mathrm{SiSr}$ & 9.83 & 8750 & 4.0 & & & & & & & & & S15 \\
\hline 379529618 & HD 12288 & $\mathrm{~A} 2 \mathrm{p} \mathrm{CrSi}$ & 7.74 & 8800 & 3.8 & & $1.6 / 8.0 /-$ & 1 & 34.9 & 20 & $\mathrm{r}$ & 1 & & $\mathrm{~S} 18,19,25$ \\
\hline 389105232 & $\mathrm{BD}+572636$ & $\mathrm{Ap} \mathrm{SrCrEu}$ & 9.55 & 7900 & 3.8 & & & & & & & & & S16,17 \\
\hline 391462523 & HD 187400 & $\mathrm{~A} 0 \mathrm{p} \mathrm{Si}$ & 8.63 & 9100 & 4.1 & & & & & & & & & $\mathrm{~S} 14$ \\
\hline 403625657 & HD 11187 & $\mathrm{~A} 0 \mathrm{p} \mathrm{SiCrSr}$ & 7.94 & 10750 & 3.5 & & $0.6 /-/-$ & & & & (s) & 3 & & S18 \\
\hline 408211646 & HD 332312 & $\mathrm{~A} 4 \mathrm{p} \mathrm{Sr}$ & 9.72 & 8650 & 3.5 & & & & & & & & & S14,15 \\
\hline 409522234 & HD 14437 & B9p CrEuSr & 7.27 & 10450 & 3.9 & & 1.9/7.6/- & 1 & 26.87 & 20 & $\mathrm{r}$ & 1 & & $\mathrm{~S} 18$ \\
\hline 430355895 & $\mathrm{BD}+523124$ & A0p Si & 10.12 & 9050 & 4.0 & roAp? & & & & & & & & S16,17 \\
\hline 450217536 & TYC 3729-816-1 & F0p SrSi & 11.35 & 6650 & 3.6 & & & & & & & & & S19 \\
\hline 468507699 & HD 206977 & A5p SrEu & 8.98 & 8650 & 3.8 & & & & & & & & & S15,16 \\
\hline 470837956 & $\mathrm{BD}+612565$ & $\mathrm{~A} 8 \mathrm{p} \mathrm{SrEuCr}$ & 9.9 & 8200 & 3.4 & roAp? & & & & & & & & $\mathrm{S} 17,18$ \\
\hline 470878580 & BD+65 1981 & $\mathrm{ApSi}$ & 10.09 & 10550 & 4.3 & roAp? & & & & & & & & $\mathrm{S} 17,18,24$ \\
\hline
\end{tabular}

References. (1) Mathys (2017); (2) Balona et al. (2011); (3) this paper; (4) Kudryavtsev \& Romanyuk (2012); (5) Romanyuk et al. (2017); (6) Romanyuk et al. (2018); (7) Balona et al. (2011); (8) Babcock (1958); (9) Preston (1971); (10) Ziznovsky \& Romanyuk (1990); (11) Aurière et al. (2007); (12) Mathys et al. (1997); (13) Hey et al. (2019); (14) Adelman (1981); (15) Pyper \& Adelman (2017); (16) Mathys et al. (2016); (17) Hümmerich et al. (2016); (18) Netopil et al. (2017); (19) Wraight et al. (2012); (20) Wade et al. (2000); (21) Zorec \& Royer (2012). 


\section{Appendix B: Amplitude spectra of long rotation period Ap star candidates}

Figures B.1 to B.9 show the amplitude spectra of those Ap stars that we have identified as having long rotation periods and that are not shown in Fig. 1.
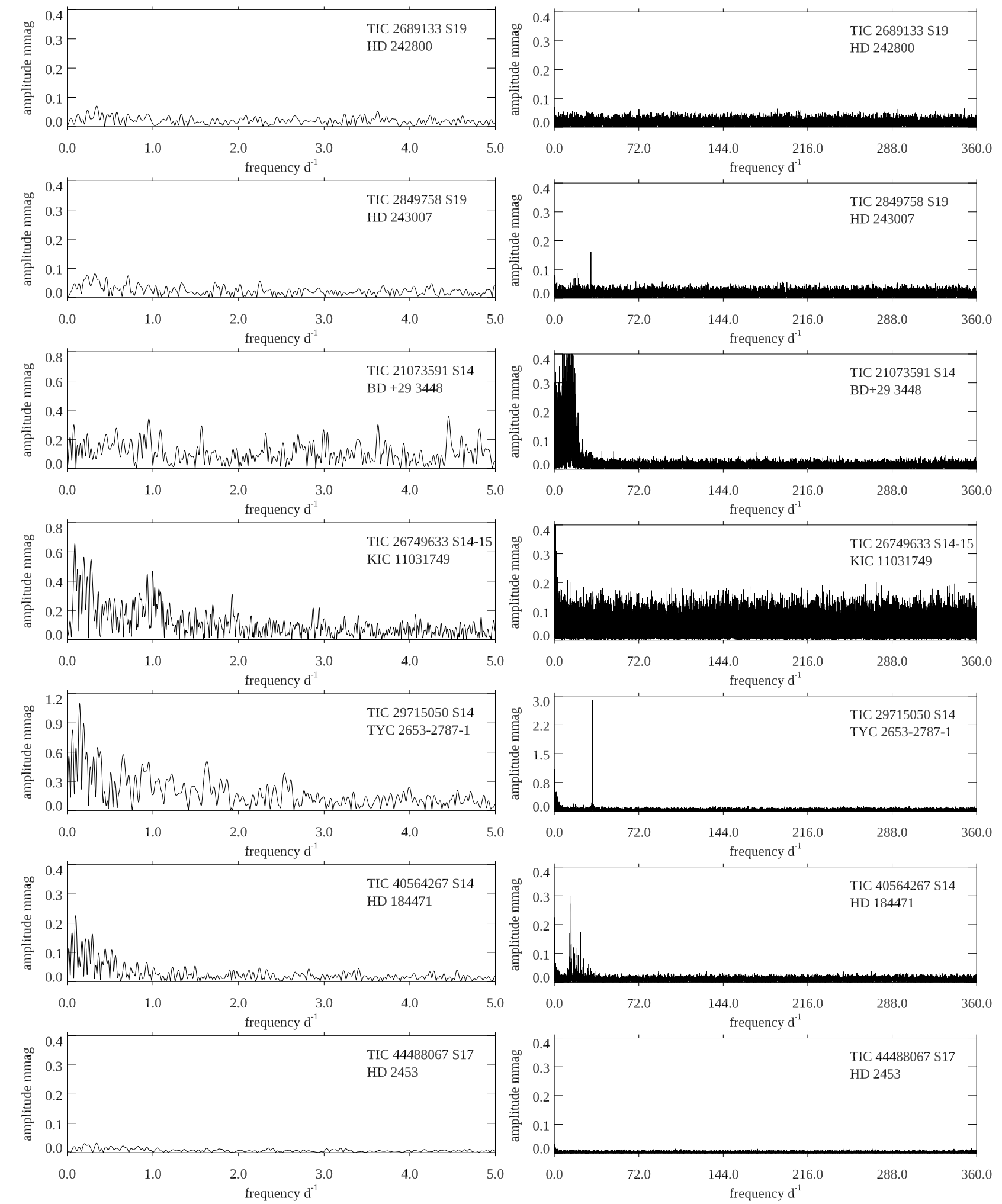

Fig. B.1. Amplitude spectra for the long-period Ap stars, continued. TIC 21073591 and TIC 40564267 are $\delta$ Sct stars (Section 2.2 and Fig. 2). TIC 29715050 is an roAp star (section 2.1). 

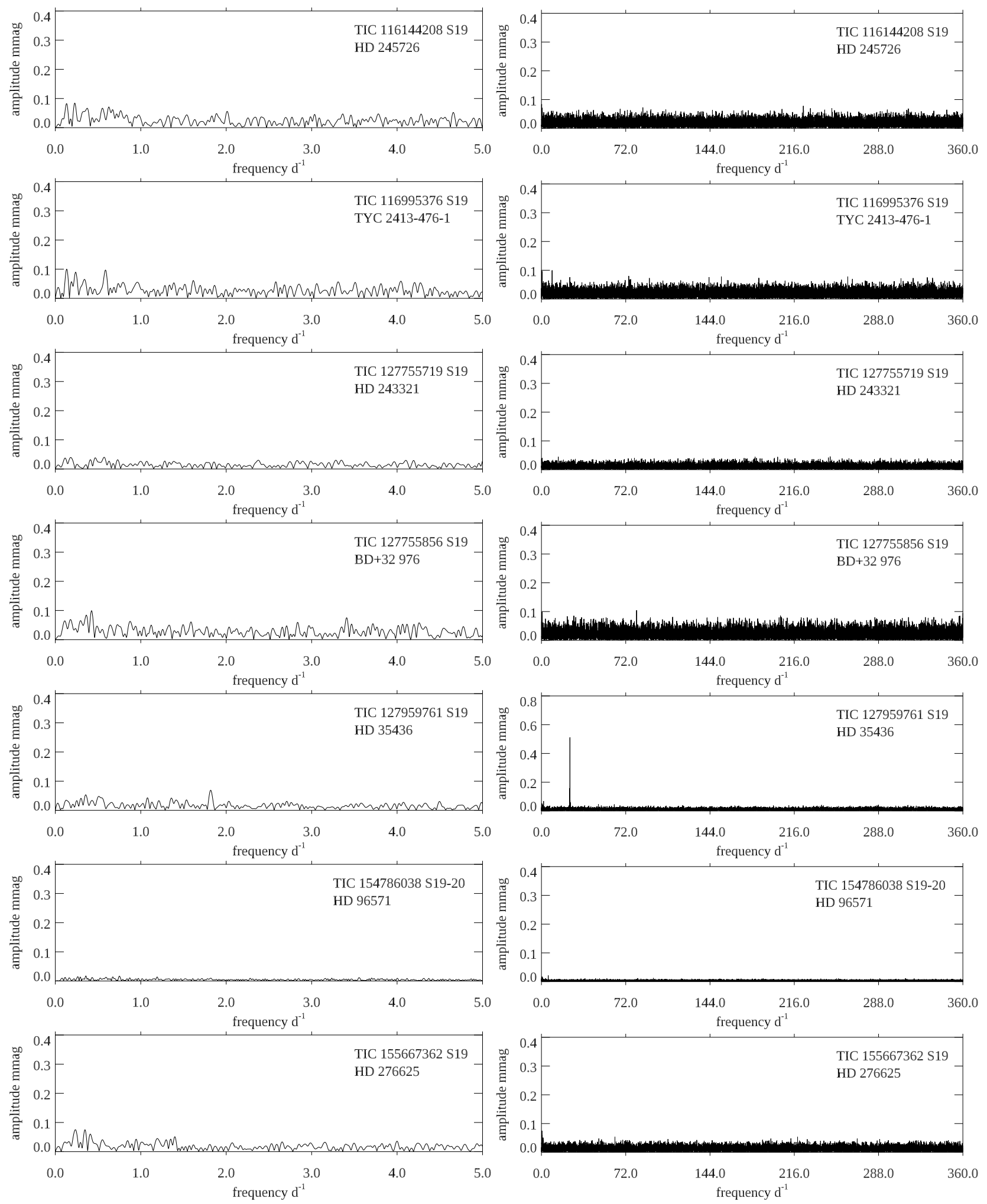

Fig. B.2. Amplitude spectra for the long-period Ap stars, continued. TIC 127959761 is a $\delta$ Sct star (Section 2.2 and Fig. 2). 


\section{G. Mathys et al.: Long period Ap stars}
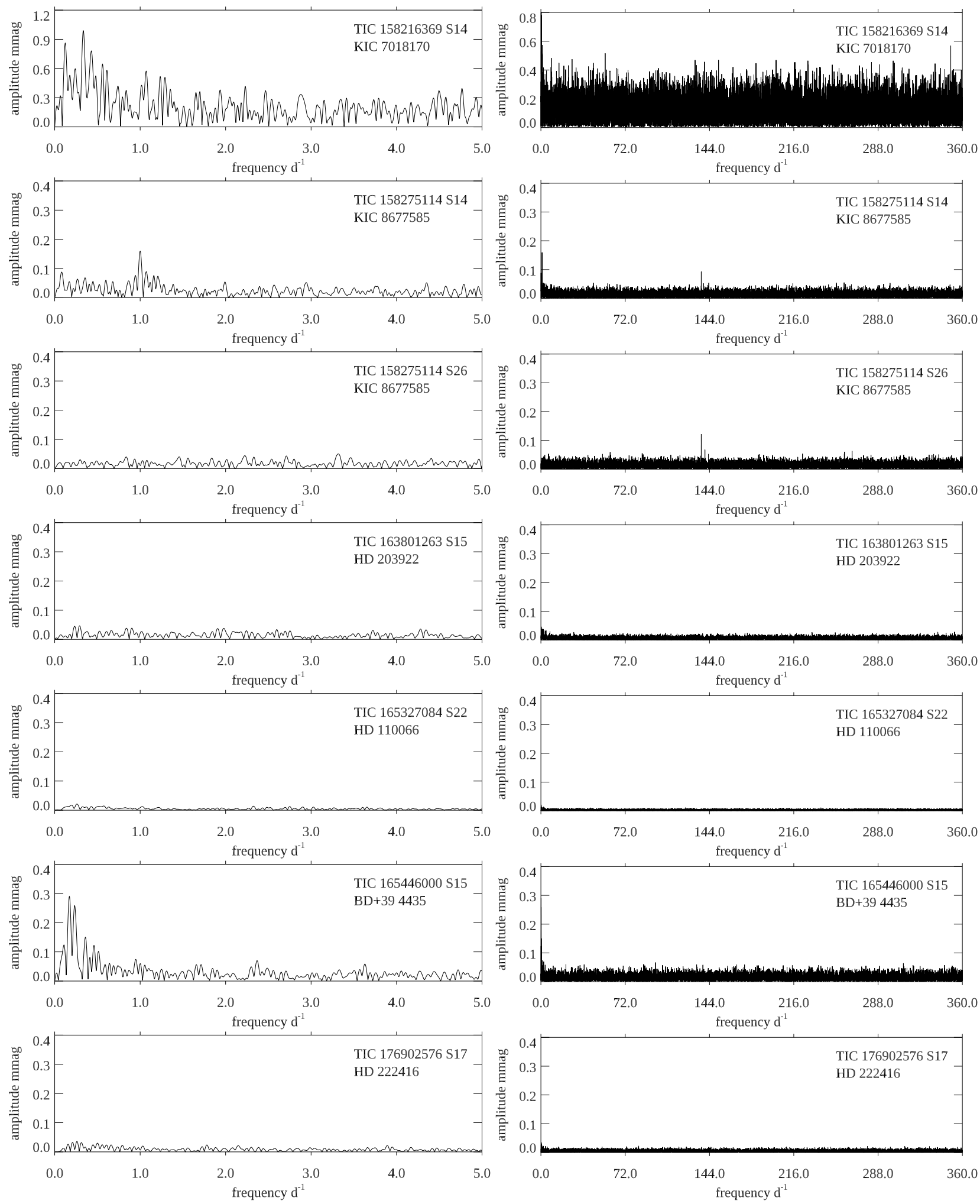

Fig. B.3. Amplitude spectra for the long-period Ap stars, continued. For TIC 158275114, S14 and S26 are plotted separately, showing that the low frequency peak near $1 \mathrm{~d}^{-1}$ is not present in both sectors, hence is not a rotation signal. This star is a well-studied roAp star, KIC 8677585 (Balona et al. 2013) (section 2.1). 

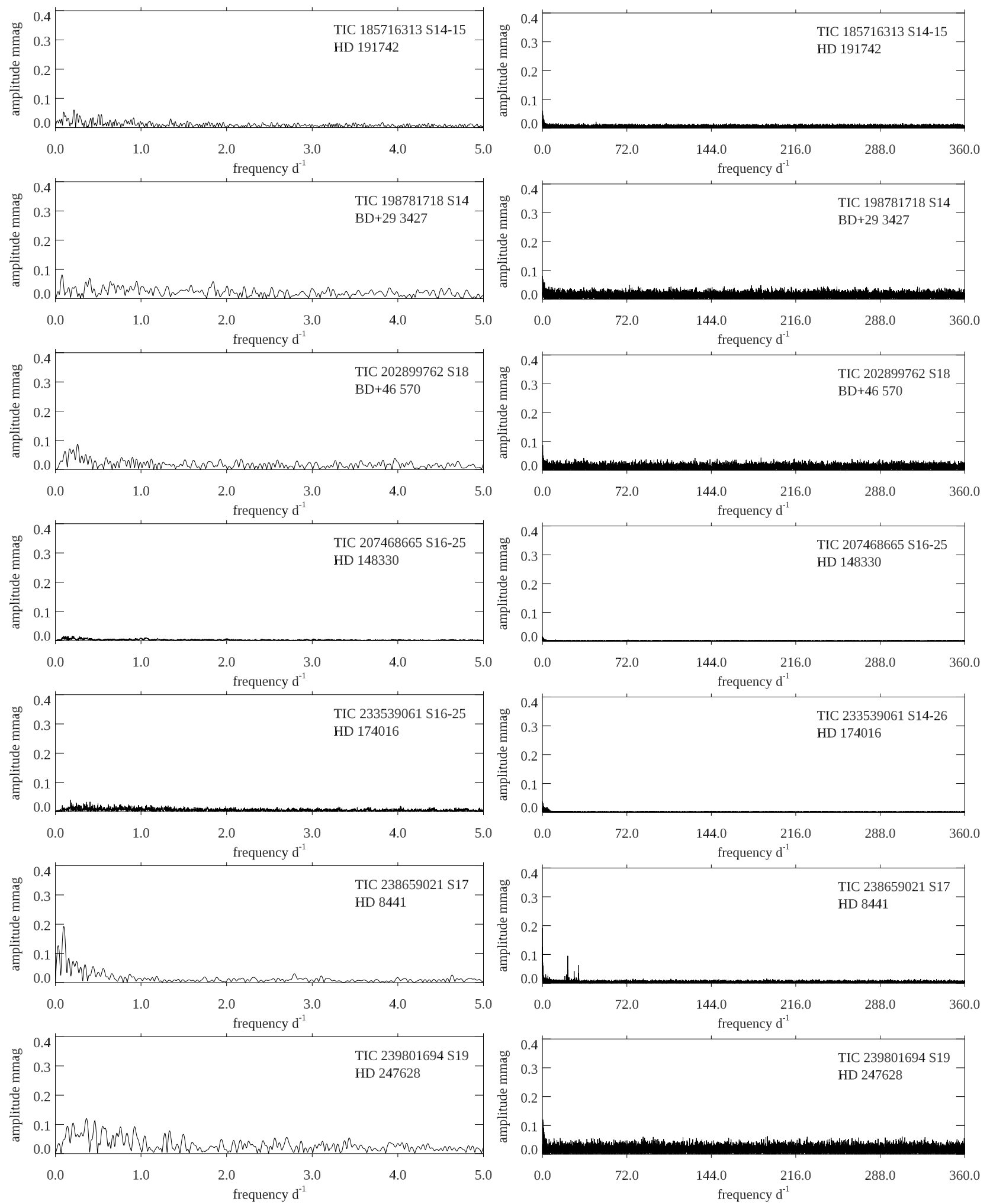

Fig. B.4. Amplitude spectra for the long-period Ap stars, continued. TIC 238659021 is a $\delta$ Sct star (Section 2.2 and Fig. 2). 
G. Mathys et al.: Long period Ap stars
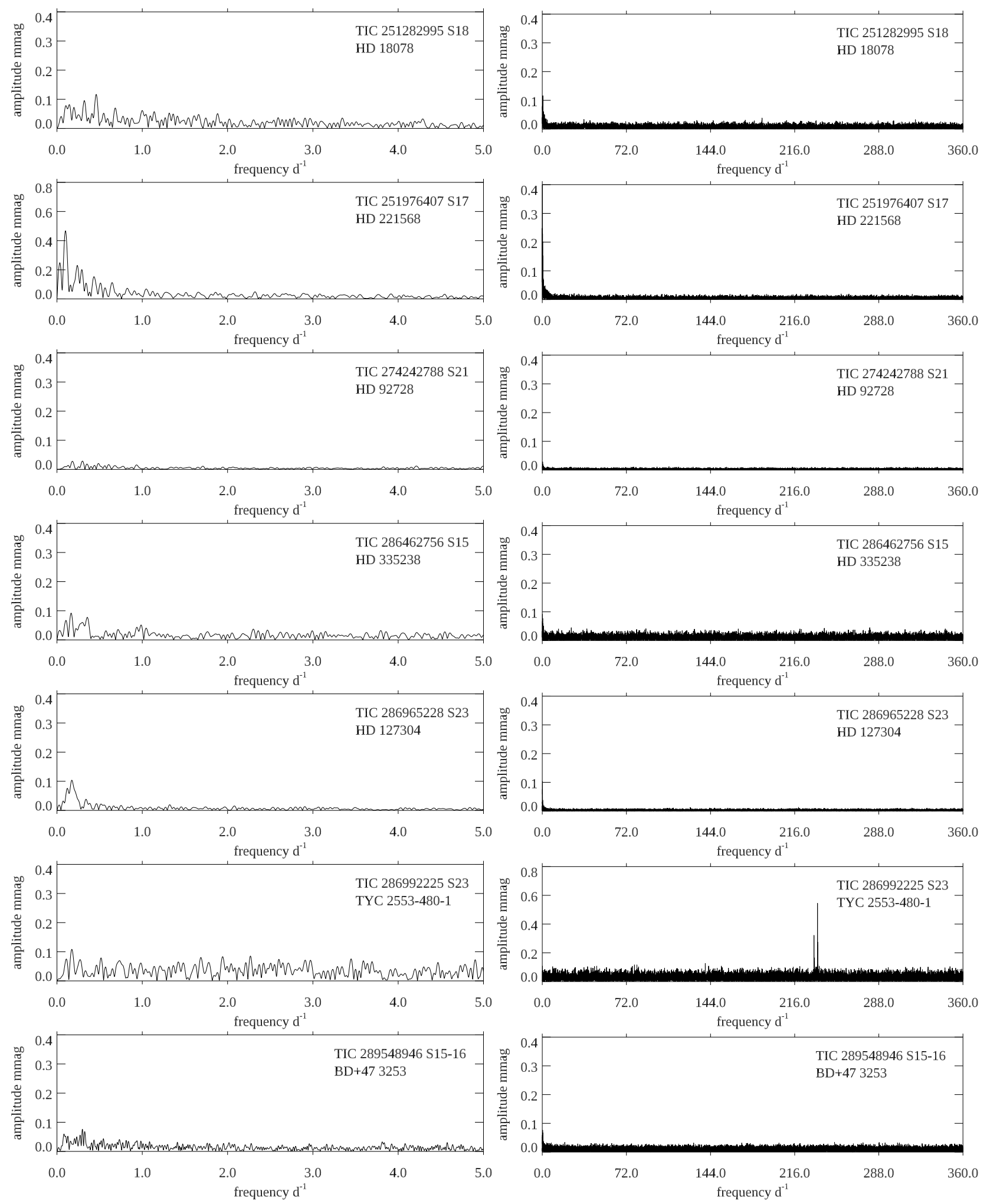

Fig. B.5. Amplitude spectra for the long-period Ap stars, continued. TIC 286992225 is an roAp star (section 2.1). 

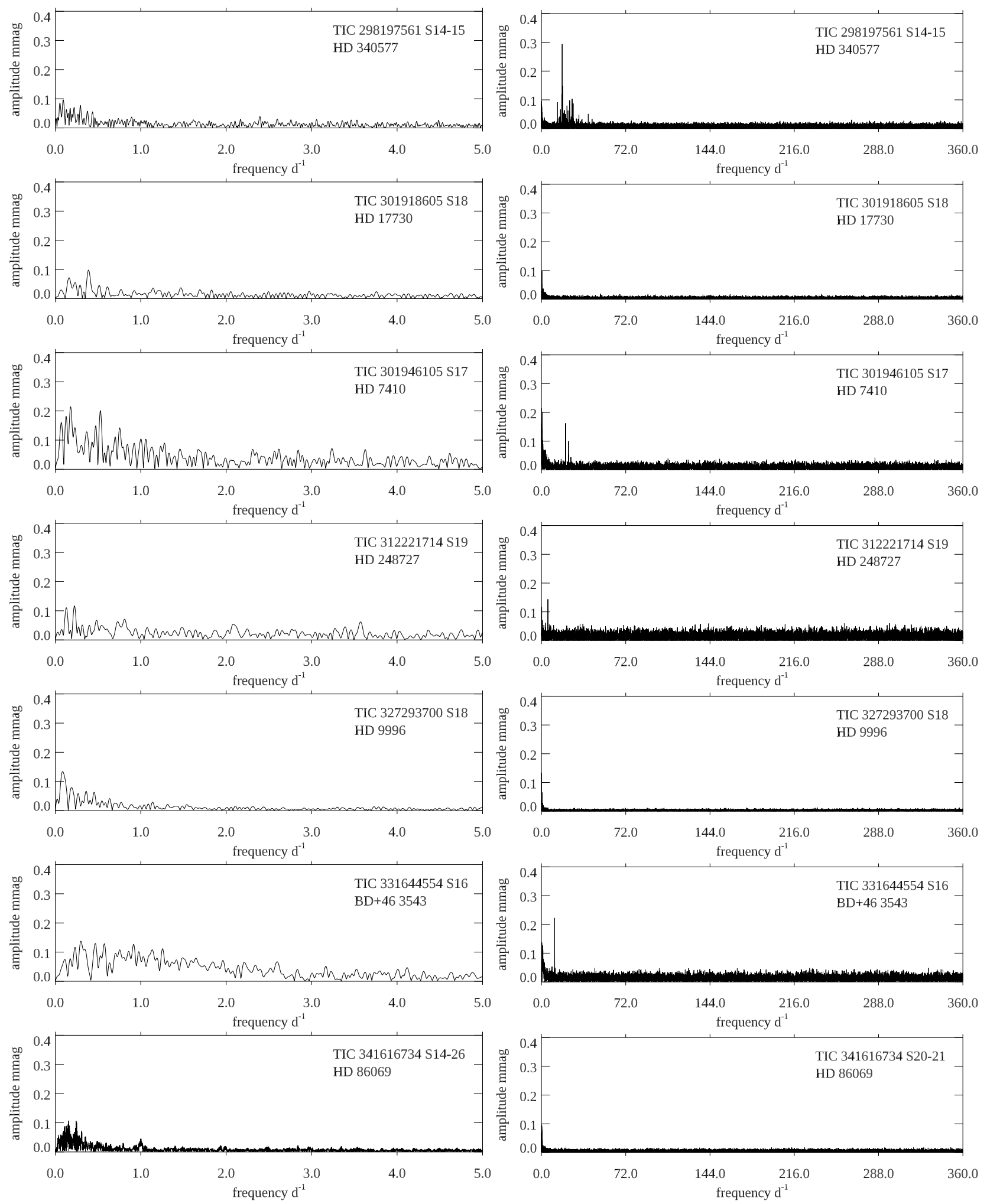

Fig. B.6. Amplitude spectra for the long-period Ap stars, continued. TIC 298197561, TIC 301918605, and TIC 331644554 are $\delta$ Sct stars (Section 2.2 and Fig. 2). TIC 312221714 has a peak at $5.5 \mathrm{~d}^{-1}$ that is between the expected frequency ranges for g modes and p modes in these stars; perhaps it is a mixed mode. 
G. Mathys et al.: Long period Ap stars
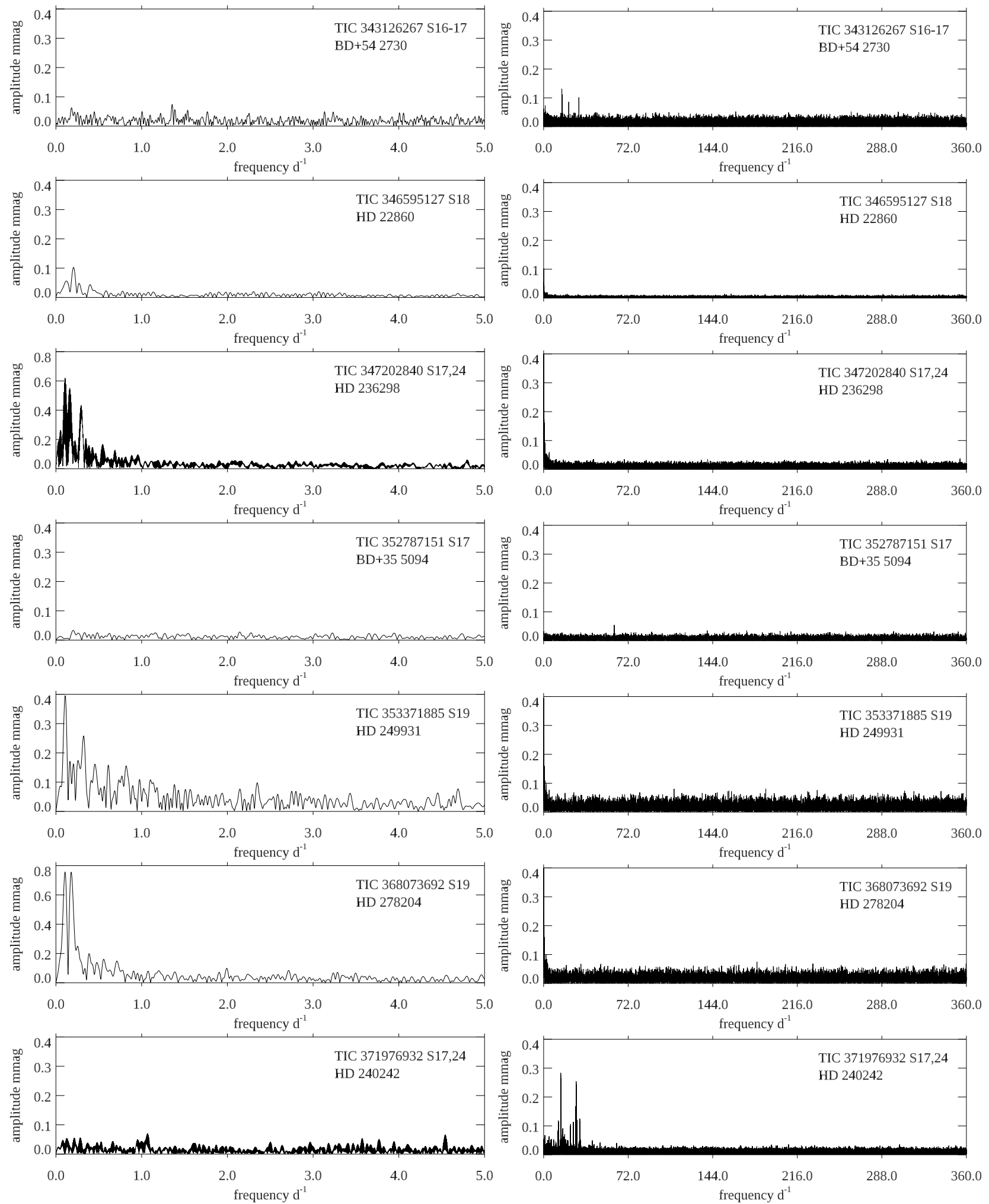

Fig. B.7. Amplitude spectra for the long-period Ap stars, continued. TIC 343126267 and TIC 371976932 are $\delta$ Sct stars (Section 2.2 and Fig. 2). TIC 352787151 is a possible roAp star (section 2.1). The unexplained low frequency variability in TIC 347202840 is different in the two sectors, hence is not periodic and is not rotational. 

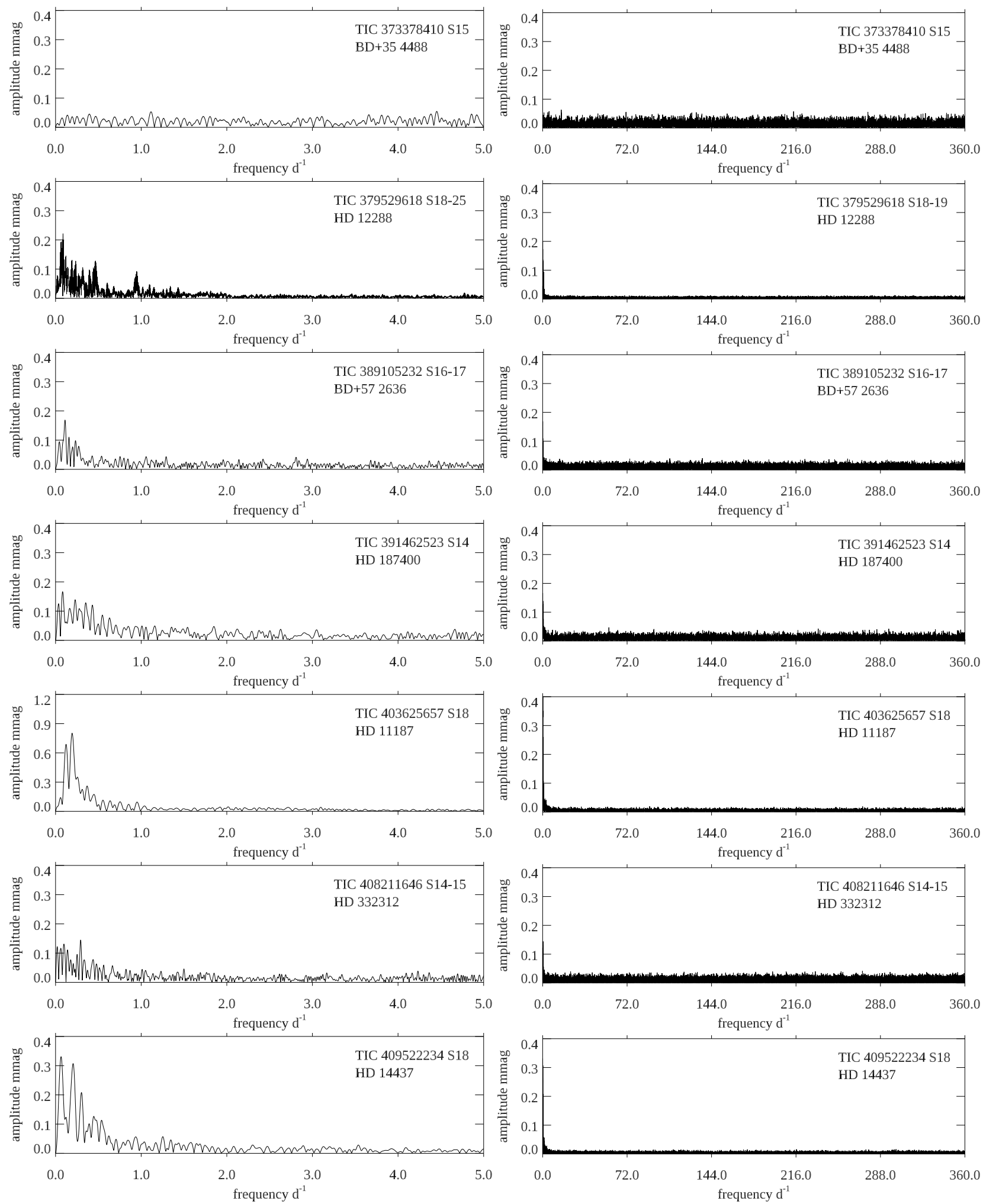

Fig. B.8. Amplitude spectra for the long-period Ap stars, continued. TIC 379529618 shows some low-frequency variability that is not rotational. The peak at $0.96 \mathrm{~d}^{-1}$ is coherent across two sectors, but does not appear in the third sector. It is of unknown origin. 
G. Mathys et al.: Long period Ap stars
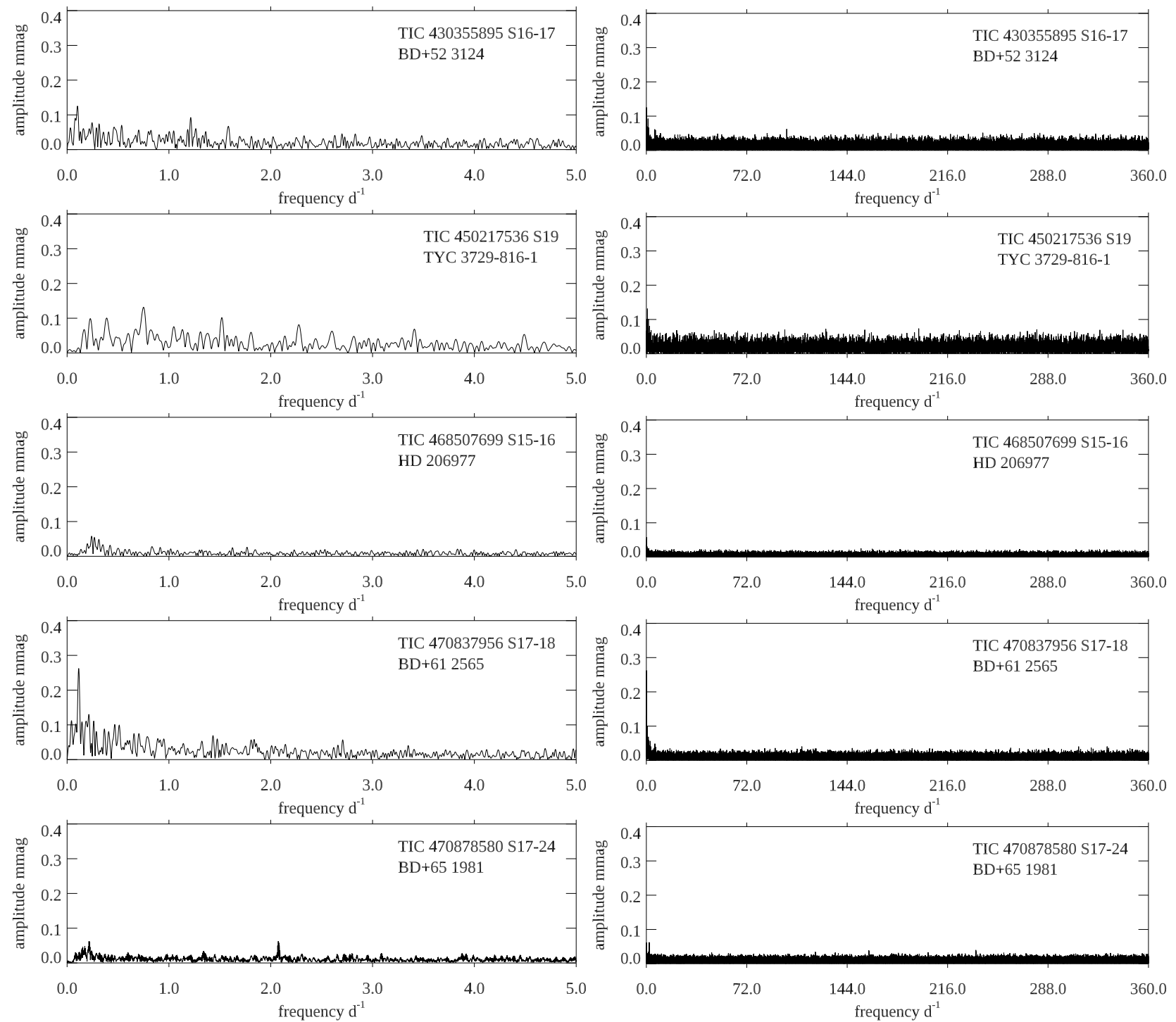

Fig. B.9. Amplitude spectra for the long-period Ap stars, continued. 